\title{
GENERICITY OF CAUSTICS AND WAVEFRONTS ON AN $r$-CORNER*
}

\author{
TAKAHARU TSUKADA ${ }^{\dagger}$
}

\begin{abstract}
We investigate genericities of reticular Lagrangian maps and reticular Legendrian maps in order to give generic classifications of caustics and wavefronts generated by a hypersurface germ without or with a boundary in a smooth manifold. We also give simpler proofs of main results in $[9],[10]$.
\end{abstract}

Key words. Caustic, wavefront, r-corner, stability, genericity.

AMS subject classifications. 58K25, 53Dxx

1. Introduction. Lagrangian and Legendrian singularities can be found in many problems of differential geometry, calculus of variations and mathematical physics. One of the most successful their applications is the study of singularities of caustics and wavefronts. For example, the particles incident along geodesics from a smooth hypersurface in a Riemannian manifold to conormal directions define a Lagrangian submanifold at a point in the cotangent bundle and define a Legendrian submanifolds at a point in the 1 -jet bundle. The caustic generated by the hypersurface is regarded as the caustic of the Lagrangian map defined by the restriction of the cotangent bundle projection to the Lagrangian submanifold and the wavefront generated by the hypersurface is regarded as the wavefront of the Legendrian map defined by the restriction of the 1-jet bundle projection to the Legendrian submanifold. Therefore the studies of the caustics and wavefronts generated by smooth hypersurfaces are reduced to the studies of Lagrangian and Legendrian singularities.
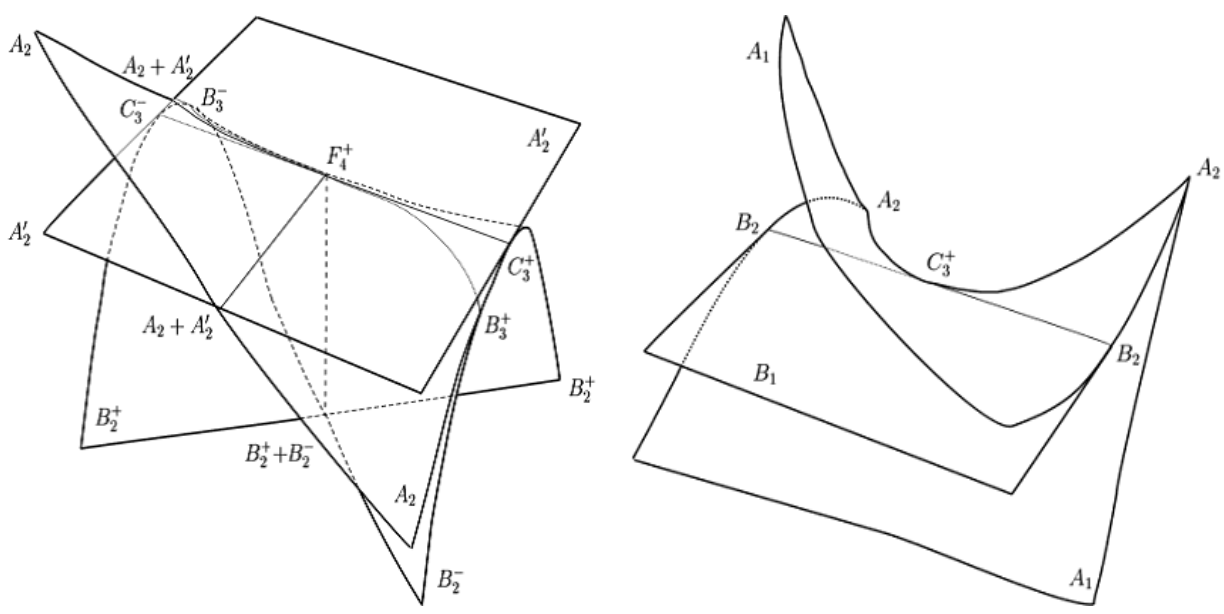

FIG. 1. the caustic $F_{4}^{+}$and the wavefront $C_{3}^{+}$

\footnotetext{
* Received May 14, 2007; accepted for publication June 18, 2010.

${ }^{\dagger}$ College of Humanities \& Sciences, Department of Mathematics, Nihon University, Higashijujo 3-1-16 Kita-ku, Tokyo 114-0001 Japan (tsukada@math.chs.nihon-u.ac.jp).
} 
In [9] and [10] we investigated the more general cases when the hypersurface has a boundary, a corner, or an r-corner. In these cases particles incident from all edges of the hypersurface give a symplectic regular $r$-cubic configuration at a point of the cotangent bundle which is a generalization of the notion of Lagrangian submanifolds and particles incident from all edges of the hypersurface give a contact regular $r$-cubic configuration at a point of the 1-jet bundle which is a generalization of the notion of Legendrian submanifolds. The caustic generated by the hypersurface germ with an $r$-corner is given by the caustic of the symplectic regular $r$-cubic configuration which is a generalization of the notion of quasicaustics given by S.Janeszko (cf., [7]). In these papers we investigated the stabilities of caustics and wavefronts generated by the hypersurface germ with an $r$-corner by studying the stabilities of reticular Lagrangian, Legendrian maps which are generalizations of the notions of Lagrangian, Legendrian maps for our situations.

In this paper, we investigate the genericities of caustics and wavefronts generated by a hypersurface with an $r$-corner. In order to realize this purpose, we shall investigate the genericities of reticular Lagrangian, Legendrian maps. In these processes, we shall need to prove the equivalences of the stabilities and the transversal stabilities of reticular Lagrangian, Legendrian maps respectively. We shall use G.Ishikawa's methods (cf., [5, Section 5]) for our situation (see also [5, Corollary 1.2]). They are simpler than the proofs of the assertions $(1) \Leftrightarrow(5)$ of Theorem 5.5 in $[9$, p.587] and Theorem 7.4 in [10, p.123] respectively.

The main results in this paper are the generic classifications of caustics generated by the hypersurface germ with an $r$-corner on an $n$ dimensional manifold in the cases $n \leq 5, r=0$ and $n \leq 3, r=1$ and wavefronts in the cases $n \leq 6, r=0$ and $n \leq 4, r=1$. In order to realize this, we shall classify generic reticular Lagrangian, Legendrian maps for the above cases.

By our theory, we have that: A generic caustics is one of the types $A_{2}, A_{3}^{ \pm}, A_{4}, A_{5}^{ \pm}, A_{6}, D_{4}^{ \pm}, D_{4}^{ \pm}, D_{6}^{ \pm}, E_{6}^{ \pm}$in the case $n \leq 5, r=0$ and $B_{2}^{ \pm}, B_{3}^{ \pm}, B_{4}^{ \pm}, C_{3}^{ \pm}, C_{4}^{ \pm}, F_{4}^{ \pm}$in the case $n \leq 3, r=1$. A generic wavefront is one of the types $A_{1}, A_{2}, A_{3}, A_{4}, A_{4}, A_{6}, D_{4}^{ \pm}, D_{5}, D_{6}^{ \pm}, E_{6}$ in the case $n \leq 6, r=0$ and $B_{2}, B_{3}, B_{4}, C_{3}^{ \pm}, C_{4}, F_{4}$ in the case $n \leq 4, r=1$.

In history, our theory in the case $r=0$ is known as Lagrangian and Legendrian singularities (cf., [2]). The important theorems (cf., [2, p.325 Theorem, p.333 Theorem]) claim the equivalences of the stabilities of Lagrangian and Legendrian maps and their generating families respectively. They are based on [1], but their proofs are omitted. G. Ishikawa has given his theory (cf., [5]) of isotropic mappings which contains the explicit proof of [2, p.325 Theorem]. He calls it Arnol'd, Tsukada theorem (cf., [5, p.125 Theorem 1.1]) because the author has given its explicit proof in [9] and he has referred it in his paper. In [9],[10], the author formulated our theory including the explicit proofs of the above theorems by the methods which are partly different from Ishikawa's method. Our theory in the case $r=1$ has been studied by I.G.Scherbak(cf., [8]). She has introduced the notion of Lagrangian andLegendrian pairs which is corresponding to the notion of regular 1-cubic configurations. But they are not strictly formulated and no proof is given. The figures of generic caustics are given in [2]. They do not distinguish between $X^{+}$and $X^{-}$types and the figures of generic wavefronts are given in neither [2] nor [8]. We give all figures of the generic caustics and wavefronts in the above cases. In the cases $r \geq 2$, our methods are not available for generic classifications because of modalities of generating families. We 
shall give an idea for a classification of generic caustics in the case $r=2$ at the last section.

This paper consists of two parts. In Part I we recall the theory of reticular Lagrangian and Legendrian maps and give some modifies for our purpose. In part 4 we investigate the genericities of reticular Lagrangian and Legendrian maps and give the generic classifications of caustics in the cases $r=0, n \leq 5$ and $r=1, n \leq 3$, wavefronts in the cases $r=0, n \leq 6$ and $r=1, n \leq 4$ respectively.

Part I. Review of reticular Lagrangian and Legendrian singularities. We give a review of [9] and [10]. Details of their definitions and theories, see each paper.

2. Stabilities of unfoldings. Let $\mathbb{H}^{r}=\left\{\left(x_{1}, \ldots, x_{r}\right) \in \mathbb{R}^{r} \mid x_{1} \geq 0, \ldots, x_{r} \geq 0\right\}$ be an $r$-corner. We denote by $\mathcal{E}\left(r ; k_{1}, s ; k_{2}\right)$ the set of all germs at 0 in $\mathbb{H}^{r} \times \mathbb{R}^{\bar{k}_{1}}$ of smooth maps $\mathbb{H}^{r} \times \mathbb{R}^{k_{1}} \rightarrow \mathbb{H}^{s} \times \mathbb{R}^{k_{2}}$ and set $\mathfrak{M}\left(r ; k_{1}, s ; k_{2}\right)=\left\{f \in \mathcal{E}\left(r ; k_{1}, s ; k_{2}\right) \mid f(0)=\right.$ $0\}$. We denote $\mathcal{E}\left(r ; k_{1}, k_{2}\right)$ for $\mathcal{E}\left(r ; k_{1}, 0 ; k_{2}\right)$ and denote $\mathfrak{M}\left(r ; k_{1}, k_{2}\right)$ for $\mathfrak{M}\left(r ; k_{1}, 0 ; k_{2}\right)$.

If $k_{2}=1$ we write simply $\mathcal{E}(r ; k)$ for $\mathcal{E}(r ; k, 1)$ and $\mathfrak{M}(r ; k)$ for $\mathfrak{M}(r ; k, 1)$. We also write $\mathcal{E}(k)$ for $\mathcal{E}(0 ; k)$ and $\mathfrak{M}(k)$ for $\mathfrak{M}(0 ; k)$. Then $\mathcal{E}(r ; k)$ is an $\mathbb{R}$-algebra in the usual way and $\mathfrak{M}(r ; k)$ is its unique maximal ideal.

Let $I_{r}=\{1,2, \ldots, r\}$ and $(x, y)=\left(x_{1}, \ldots, x_{r}, y_{1}, \ldots, y_{k}\right)$ be a fixed coordinate system of $\left(\mathbb{H}^{r} \times \mathbb{R}^{k}, 0\right)$. We denote by $\mathcal{B}(r ; k)$ the group of diffeomorphism germs on $\left(\mathbb{H}^{r} \times \mathbb{R}^{k}, 0\right)$ of the form:

$$
\phi(x, y)=\left(x_{1} \phi_{1}^{1}(x, y), \ldots, x_{r} \phi_{1}^{r}(x, y), \phi_{2}^{1}(x, y), \ldots, \phi_{2}^{k}(x, y)\right) .
$$

We also denote by $\mathcal{B}_{n}(r ; k+n)$ the group of diffeomorphism germs on $\left(\mathbb{H}^{r} \times \mathbb{R}^{k+n}, 0\right)$ of the form:

$$
\begin{aligned}
& \phi(x, y, u) \\
& =\left(x_{1} \phi_{1}^{1}(x, y, u), \ldots, x_{r} \phi_{1}^{r}(x, y, u), \phi_{2}^{1}(x, y, u), \ldots, \phi_{2}^{k}(x, y, u), \phi_{3}^{1}(u), \ldots, \phi_{3}^{n}(u)\right) .
\end{aligned}
$$

We denote $J^{l}(r+k, 1)$ the set of $l$-jets at 0 of germs in $\mathfrak{M}(r ; k)$ and let $\pi_{l}: \mathfrak{M}(r ; k) \rightarrow J^{l}(r+k, 1)$ be the natural projection. We denote $j^{l} f(0)$ the $l$-jet of $f \in \mathfrak{M}(r ; k)$. We also denote $\phi(x, y, u)=\left(x \phi_{1}(x, y, u), \phi_{2}(x, y, u), \phi_{3}(u)\right)$ and denote other notations analogously. In this paper all maps and all map germs are supposed to be smooth.

We say that $f, g \in \mathcal{E}(r ; k)$ are reticular $\mathcal{R}$-equivalent if there exists $\phi \in \mathcal{B}(r ; k)$ such that $g=f \circ \phi$. We say that $f, g \in \mathcal{E}(r ; k)$ are reticular $\mathcal{K}$-equivalent if there exist $\phi \in \mathcal{B}(r ; k)$ and a unit $a \in \mathcal{E}(r ; k)$ such that $g=a \cdot f \circ \phi$.

We recall the stabilities of unfoldings under the reticular $\mathcal{R}^{+}(\mathcal{K})$-equivalences which are developed in [9] and [10]. In order to distinguish equivalence relations between function germs in $\mathfrak{M}(r ; k)$ and their unfoldings, we denote this equivalence relation by the reticular $\mathcal{P}-\mathcal{R}^{+}(\mathcal{K})$-equivalence in this paper.

We call a function germ $f \in \mathfrak{M}(r ; k)$ is $\mathcal{R}(\mathcal{K})$-simple if the following holds: For a sufficiently larger integer $l$, there exists a neighborhood $N$ of $j^{l} f(0)$ in $J^{l}(r+k, 1)$ such that $N$ intersects with a finite number of $\mathcal{R}(\mathcal{K})$-orbits. By [2, §17.4,p.279] we have that: 
TheOREM 2.1. An $\mathcal{R}$-simple function germ in $\mathfrak{M}(1 ; k)^{2}$ is stably $\mathcal{R}$-equivalent to one of the following function germs:

$$
B_{l}^{ \pm}: \pm x^{l}(l \geq 2), \quad C_{l}^{ \pm}: x y \pm y^{l}(l \geq 3), \quad F_{4}^{ \pm}: \pm x^{2}+y^{3} .
$$

Theorem 2.2. (cf., [10, p.123]) A $\mathcal{K}$-simple function germ in $\mathfrak{M}(1 ; k)^{2}$ is stably $\mathcal{K}$-equivalent to one of the following function germs:

$$
B_{l}: x^{l}(l \geq 2), \quad C_{l}^{\varepsilon}: x y+\varepsilon y^{l}\left(\varepsilon^{l-1}=1, l \geq 3\right), \quad F_{4}: x^{2}+y^{3} .
$$

Theorem 2.3. (cf., [9, Theorem 4.5],[10, Theorem 6.5]) Let $\mathcal{G}=\mathcal{R}^{+}$or $\mathcal{K}$ and $F \in \mathfrak{M}(r ; k+n)$ be an unfolding of $f \in \mathfrak{M}(r ; k)$. Then the following are all equivalent.

(1) $F$ is reticular $\mathcal{P}$ - $\mathcal{G}$-stable.

(2) $F$ is reticular $\mathcal{P}$-G-versal.

(3) $F$ is reticular $\mathcal{P}-\mathcal{G}$-infinitesimally versal.

(4) $F$ is reticular $\mathcal{P}$ - $\mathcal{G}$-infinitesimally stable.

(5) $F$ is reticular $\mathcal{P}$ - $\mathcal{G}$-homotopically stable.

3. Reticular Lagrangian singularity. We define $L_{\sigma}^{0}=\{(q, p) \in$ $\left.\left(T^{*} \mathbb{R}^{n}, 0\right) \mid q_{\sigma}=p_{I_{r}-\sigma}=q_{r+1}=\cdots=q_{n}=0, q_{I_{r}-\sigma} \geq 0\right\}$ for $\sigma \subset I_{r}$. Let $\mathbb{L}=\left\{(q, p) \in T^{*} \mathbb{R}^{n} \mid q_{1} p_{1}=\cdots=q_{r} p_{r}=q_{r+1}=\cdots=q_{n}=0, q_{I_{r}} \geq 0\right\}$ be the representative as a germ of the union of $L_{\sigma}^{0}$ for $\sigma \subset I_{r}$. We call a map germ

$$
(\mathbb{L}, 0) \stackrel{i}{\longrightarrow}\left(T^{*} \mathbb{R}^{n}, 0\right) \stackrel{\pi}{\longrightarrow}\left(\mathbb{R}^{n}, 0\right)
$$

a reticular Lagrangian map if there exists a symplectic diffeomorphism germ $S$ on $\left(T^{*} \mathbb{R}^{n}, 0\right)$ such that $i=\left.S\right|_{\mathbb{L}}$.

We call a symplectic diffeomorphism germ $\phi$ on $\left(T^{*} \mathbb{R}^{n}, 0\right)$ a reticular diffeomorphism if $\phi\left(L_{\sigma}^{0}\right)=L_{\sigma}^{0}$ for $\sigma \subset I_{r}$. We say that reticular Lagrangian maps $\pi \circ i_{1}, \pi \circ i_{2}:(\mathbb{L}, 0) \rightarrow\left(T^{*} \mathbb{R}^{n}, 0\right) \rightarrow\left(\mathbb{R}^{n}, 0\right)$ are Lagrangian equivalent if there exist a reticular diffeomorphism $\phi$ and a Lagrangian equivalence $\Theta$ of $\pi$ such that the following diagram is commutative:

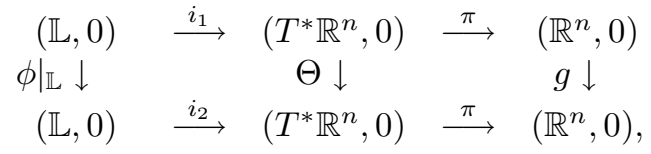

where $g$ is the diffeomorphism of the base space of $\pi$ induced by $\Theta$.

We remark that there is not the condition that a reticular diffeomorphism is a symplectic diffeomorphism in the definition in [9]. But a reticular diffeomorphism defined in [9] consists of a restriction to $\mathbb{L}$ of compositions of two symplectic diffeomorphism and a Lagrangian equivalence, it follows that a reticular diffeomorphism in $[9]$ is automatically a restriction to $\mathbb{L}$ of symplectic diffeomorphism. So our definition is equivalent to the one in [9].

We say that a function germ $H$ on $\left(T^{*} \mathbb{R}^{n}, 0\right)$ is fiber preserving if $H$ has the form $H(q, p)=\sum_{j=1}^{n} h_{j}(q) p_{j}+h_{0}(q)$.

We recall the following theorem which is proved in [9]. 
Theorem 3.1. (cf., [9, p.587 Theorem 5.5]) Let $\pi \circ i:(\mathbb{L}, 0) \rightarrow\left(T^{*} \mathbb{R}^{n}, 0\right) \rightarrow$ $\left(\mathbb{R}^{n}, 0\right)$ be a reticular Lagrangian map with a generating family $F(x, y, q) \in \mathfrak{M}(r ; k+$ $n)^{2}$. Then the following are all equivalent.

(u) $F$ is a reticular $\mathcal{R}^{+}$-stable unfolding of $\left.F\right|_{q=0}$.

(hs) $\pi \circ i$ is homotopically stable.

(is) $\pi \circ i$ is infinitesimally stable.

(a) For any function germ $f$ on $\left(T^{*} \mathbb{R}^{n}, 0\right)$, there exists a fiber preserving function germ $H$ on $\left(T^{*} \mathbb{R}^{n}, 0\right)$ such that $f \circ i=H \circ i$.

(s) $\pi \circ i$ is stable.

The definition of the infinitesimally stability of reticular Lagrangian maps in [9] seem to be different from our one. But these are equivalent by Lemma 6.1. The assertion that (u), (hs),(is) and (a) are all equivalent is proved in [9]. But the proof of $(\mathrm{u}) \Leftrightarrow(\mathrm{s})$ is slightly complicated. So we shall prove this assertion by another method in Section 6.

4. Reticular Legendrian singularity. We define $\tilde{L}_{\sigma}^{0}=\{(q, z, p) \in$ $\left.\left(J^{1}\left(\mathbb{R}^{n}, \mathbb{R}\right), 0\right) \mid q_{\sigma}=p_{I_{r}-\sigma}=q_{r+1}=\cdots=q_{n}=z=0, q_{I_{r}-\sigma} \geq 0\right\}$ for each $\sigma \subset I_{r}$. Let $\tilde{\mathbb{L}}=\left\{(q, z, p) \in J^{1}\left(\mathbb{R}^{n}, \mathbb{R}\right) \mid q_{1} p_{1}=\cdots=q_{r} p_{r}=q_{r+1}=\cdots=q_{n}=z=0, q_{I_{r}} \geq 0\right\}$ be a representative as a germ of the union of $\tilde{L}_{\sigma}^{0}$ for all $\sigma \subset I_{r}$. We call a map germ

$$
(\tilde{\mathbb{L}}, 0) \stackrel{i}{\longrightarrow}\left(J^{1}\left(\mathbb{R}^{n}, \mathbb{R}\right), 0\right) \stackrel{\tilde{\pi}}{\longrightarrow}\left(\mathbb{R}^{n} \times \mathbb{R}, 0\right)
$$

a reticular Legendrian map if there exists a contact diffeomorphism germ $C$ on $\left(J^{1}\left(\mathbb{R}^{n}, \mathbb{R}\right), 0\right)$ such that $i=\left.C\right|_{\tilde{L}}$.

We define the equivalence relation of reticular Legendrian maps. We call a contact diffeomorphism germ $\phi$ on $\left(J^{1}\left(\mathbb{R}^{n}, \mathbb{R}\right), 0\right)$ a reticular diffeomorphism if $\phi\left(\tilde{L}_{\sigma}^{0}\right)=\tilde{L}_{\sigma}^{0}$ for $\sigma \subset I_{r}$. We say that reticular Legendrian maps $\tilde{\pi} \circ i_{1}, \tilde{\pi} \circ i_{2}:(\tilde{\mathbb{L}}, 0) \rightarrow\left(J^{1}\left(\mathbb{R}^{n}, \mathbb{R}\right), 0\right) \rightarrow$ $\left(\mathbb{R}^{n} \times \mathbb{R}, 0\right)$ are Legendrian equivalent if there exist a reticular diffeomorphism $\phi$ and a Legendrian equivalence $\Theta$ of $\tilde{\pi}$ such that the following diagram is commutative:

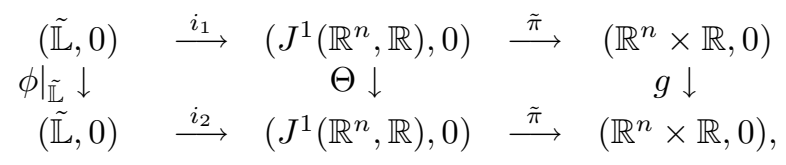

where $g$ is the diffeomorphism of the base space of $\tilde{\pi}$ induced by $\Theta$.

We say that a function germ $H$ on $\left(J^{1}\left(\mathbb{R}^{n}, \mathbb{R}\right), 0\right)$ is fiber preserving if $H$ has the form $H(q, z, p)=\sum_{j=1}^{n} h_{j}(q, z) p_{j}+h_{0}(q, z)$.

TheOREM 4.1. (cf., [10, p.123 Theorem 7.4]) Let $\tilde{\pi} \circ i:(\tilde{\mathbb{L}}, 0) \rightarrow\left(J^{1}\left(\mathbb{R}^{n}, \mathbb{R}\right), 0\right) \rightarrow$ $\left(\mathbb{R}^{n} \times \mathbb{R}, 0\right)$ be a reticular Legendrian map with a generating family $F(x, y, q, z) \in$ $\mathfrak{M}(r ; k+n+1)$. Then the following are all equivalent.

(u) $F$ is a reticular $\mathcal{P}-\mathcal{K}$-stable unfolding of $\left.F\right|_{q=z=0}$.

(hs) $\tilde{\pi} \circ i$ is homotopically stable.

(is) $\tilde{\pi} \circ i$ is infinitesimally stable.

(a) For any function germ $f$ on $\left(J^{1}\left(\mathbb{R}^{n}, \mathbb{R}\right), 0\right)$, there exists a fiber preserving function germ $H$ on $\left(J^{1}\left(\mathbb{R}^{n}, \mathbb{R}\right), 0\right)$ such that $f \circ i=H \circ i$.

(s) $\tilde{\pi} \circ i$ is stable.

We shall prove the assertion $(\mathrm{u}) \Leftrightarrow(\mathrm{s})$ by the simpler method than that of $[10]$ in Section 7 . 
Part II. Genericities of reticular Lagrangian, Legendrian maps. In this part, we shall investigate finite determinacies of reticular Lagrangian and Legendrian maps. We shall reduce our investigation to finite dimensional jet spaces of symplectic and contact diffeomorphism germs and give the generic classifications of caustics and wavefronts in the cases $r=0, r=1$ respectively.

5. Finite determinacy of reticular Lagrangian, Legendrian maps. DEFINITION 5.1. Let $\pi \circ i:(\mathbb{L}, 0) \rightarrow\left(T^{*} \mathbb{R}^{n}, 0\right) \rightarrow\left(\mathbb{R}^{n}, 0\right)$ be a reticular Lagrangian map and $l$ be a non-negative number. We say that $\pi \circ i$ is $l$-determined if the following condition holds: For any extension $S$ of $i$, the reticular Lagrangian map $\left.\pi \circ S^{\prime}\right|_{\mathbb{L}}$ and $\pi \circ i$ are Lagrangian equivalent for any symplectic diffeomorphism germ $S^{\prime}$ on $\left(T^{*} \mathbb{R}^{n}, 0\right)$ satisfying $j^{l} S(0)=j^{l} S^{\prime}(0)$.

Lemma 5.2. Let $\pi \circ i:(\mathbb{L}, 0) \rightarrow\left(T^{*} \mathbb{R}^{n}, 0\right) \rightarrow\left(\mathbb{R}^{n}, 0\right)$ be a reticular Lagrangian map. Let $S_{1}, S_{2}$ be symplectic diffeomorphism germs on $\left(T^{*} \mathbb{R}^{n}, 0\right)$ such that $\left.S_{1}\right|_{\mathbb{L}}=$ $\left.S_{2}\right|_{\mathbb{L}}=i$. Then there exists a reticular diffeomorphism germ $\phi$ such that $S_{1}=S_{2} \circ \phi$.

Proof. Set $\phi=\left(S_{2}\right)^{-1} \circ S_{1}$. Then we have that $\left.\phi\right|_{\mathbb{L}}=i d$ and $S_{1}=S_{2} \circ \phi$.

By this lemma we have that the finite determinacy of reticular Lagrangian maps do not depend on choices of extensions of their reticular Lagrangian embeddings.

Theorem 5.3. Let $\pi \circ i:(\mathbb{L}, 0) \rightarrow\left(T^{*} \mathbb{R}^{n}, 0\right) \rightarrow\left(\mathbb{R}^{n}, 0\right)$ be a reticular Lagrangian map. If $\pi \circ i$ is infinitesimally stable then $\pi \circ i$ is $(n+1)$-determined.

Proof. Let $S$ be an extension of $i$. Since the infinitesimally stability of reticular Lagrangian maps is invariant under Lagrangian equivalences, we may assume that the canonical relation $P_{S}$ associated with $S$ has the form:

$$
P_{S}=\left\{\left(Q,-\frac{\partial H}{\partial Q}(Q, p),-\frac{\partial H}{\partial p}(Q, p), p\right) \in\left(T^{*} \mathbb{R}^{n} \times T^{*} \mathbb{R}^{n},(0,0)\right)\right\}
$$

for some function germ $H(Q, p) \in \mathfrak{M}(2 n)^{2}$. Then the function germ $F(x, y, q)=$ $H_{0}(x, y)+\langle y, q\rangle \in \mathfrak{M}(r ; n+n)^{2}$ is a generating family of $\pi \circ i$, where $H_{0}(x, y)=$ $H\left(x_{1}, \ldots, x_{r}, 0, \ldots, 0, y_{1}, \ldots, y_{n}\right) \in \mathfrak{M}(r ; n)^{2}$. Since $\pi \circ i$ is infinitesimally stable, it follows that $F$ is a reticular $\mathcal{P}$ - $\mathcal{R}^{+}$-infinitesimally versal unfolding of $H_{0}(x, y)$ by Theorem 3.1. This means that $\mathcal{E}(r ; n) /\left\langle x \frac{\partial H_{0}}{\partial x}, \frac{\partial H_{0}}{\partial y}\right\rangle_{\mathcal{E}(r ; n)}$ is at most $(n+1)$-dimension. It follows that

$$
\mathfrak{M}(r ; n)^{n+1} \subset\left\langle x \frac{\partial H_{0}}{\partial x}, \frac{\partial H_{0}}{\partial y}\right\rangle_{\mathcal{E}(r ; n)} .
$$

Therefore we have that

$$
\mathfrak{M}(r ; n)^{n+3} \subset \mathfrak{M}(r ; n)\left(\left\langle x \frac{\partial H_{0}}{\partial x}\right\rangle+\mathfrak{M}(r ; n)\left\langle\frac{\partial H_{0}}{\partial y}\right\rangle\right) .
$$

This means that $H_{0}$ is reticular $\mathcal{R}$ - $(n+2)$-determined by [9, p.583, Lemma 4.1]. Let a symplectic diffeomorphism germ $S^{\prime}$ on $\left(T^{*} \mathbb{R}^{n}, 0\right)$ satisfying $j^{n+1} S(0)=j^{n+1} S^{\prime}(0)$ be given. Since $\frac{\partial p \circ S}{\partial p}(0)=\frac{\partial p \circ S^{\prime}}{\partial p}(0)$, it follows that there exists a function germ $H^{\prime}(Q, p) \in \mathfrak{M}(2 n)^{2}$ such that

$$
P_{S^{\prime}}=\left\{\left(Q,-\frac{\partial H^{\prime}}{\partial Q}(Q, p),-\frac{\partial H^{\prime}}{\partial p}(Q, p), p\right)\right\} .
$$


Then the function germ $G(x, y, q):=H_{0}^{\prime}(x, y)+\langle y, q\rangle \in \mathfrak{M}(r ; n+n)^{2}$ is a generating family of $\left.\pi \circ S^{\prime}\right|_{\mathbb{L}}$, where $H_{0}^{\prime}(x, y)=H^{\prime}(x, 0, y) \in \mathfrak{M}(r ; n)^{2}$. Since $j^{n+1} S(0)=$ $j^{n+1} S^{\prime}(0)$, it means that $j^{n+1} \frac{\partial H}{\partial Q}(0)=j^{n+1} \frac{\partial H^{\prime}}{\partial Q}(0), j^{n+1} \frac{\partial H}{\partial p}(0)=j^{n+1} \frac{\partial H^{\prime}}{\partial p}(0)$. Therefore we have that $j^{n+2} H(0)=j^{n+2} H^{\prime}(0)$ and hence $j^{n+2} H_{0}(0)=j^{n+2} H_{0}^{\prime}(0)$. Thus there exists $\Phi(x, y) \in \mathcal{B}(r ; n)$ such that $H_{0}=H_{0}^{\prime} \circ \Phi$. We set $G^{\prime}(x, y, q):=$ $G(\Phi(x, y), q) \in \mathfrak{M}(r ; n+n)^{2}$. Then $G$ and $G^{\prime}$ are reticular $\mathcal{P}$ - $\mathcal{R}$-equivalent, and $F$ and $G^{\prime}$ are reticular $\mathcal{P}-\mathcal{R}^{+}$-infinitesimal versal unfoldings of $H_{0}(x, y)$. It follows that $F$ and $G$ are reticular $\mathcal{P}$ - $\mathcal{R}^{+}$-equivalent. Therefore $\pi \circ i$ and $\left.\pi \circ S^{\prime}\right|_{\mathbb{L}}$ are Lagrangian equivalent.

We consider contact diffeomorphism germs on $\left(J^{1}\left(\mathbb{R}^{n}, \mathbb{R}\right), 0\right)$. Let $(Q, Z, P)$ be canonical coordinates on the source space and $(q, z, p)$ be canonical coordinates of the target space. We define the following notations:

$\imath:\left(J^{1}\left(\mathbb{R}^{n}, \mathbb{R}\right) \cap\{Z=0\}, 0\right) \rightarrow\left(J^{1}\left(\mathbb{R}^{n}, \mathbb{R}\right), 0\right)$ be the inclusion map on the source space.

$C\left(J^{1}\left(\mathbb{R}^{n}, \mathbb{R}\right), 0\right)=\left\{C:\left(J^{1}\left(\mathbb{R}^{n}, \mathbb{R}\right), 0\right) \rightarrow\left(J^{1}\left(\mathbb{R}^{n}, \mathbb{R}\right), 0\right) \mid C:\right.$ contact diffeomorphism $\}$ $C^{\theta}\left(J^{1}\left(\mathbb{R}^{n}, \mathbb{R}\right), 0\right)=\left\{C \in C\left(J^{1}\left(\mathbb{R}^{n}, \mathbb{R}\right), 0\right) \mid C\right.$ preserves the canonical 1-form $\}$

$C_{Z}\left(J^{1}\left(\mathbb{R}^{n}, \mathbb{R}\right), 0\right)=\left\{C \circ \imath \mid C \in C\left(J^{1}\left(\mathbb{R}^{n}, \mathbb{R}\right), 0\right)\right\}$

$C_{Z}^{\theta}\left(J^{1}\left(\mathbb{R}^{n}, \mathbb{R}\right), 0\right)=\left\{C \circ \imath \mid C \in C^{\theta}\left(J^{1}\left(\mathbb{R}^{n}, \mathbb{R}\right), 0\right)\right\}$.

Definition 5.4. Let $\tilde{\pi} \circ i:(\tilde{\mathbb{L}}, 0) \rightarrow\left(J^{1}\left(\mathbb{R}^{n}, \mathbb{R}\right), 0\right) \rightarrow\left(\mathbb{R}^{n} \times \mathbb{R}, 0\right)$ be a reticular Legendrian map. We say that $\tilde{\pi} \circ i$ is $l$-determined if the following condition holds: For any extension $C \in C\left(J^{1}\left(\mathbb{R}^{n}, \mathbb{R}\right), 0\right)$ of $i$, the reticular Legendrian map $\left.\tilde{\pi} \circ C^{\prime}\right|_{\tilde{\mathbb{L}}}$ and $\tilde{\pi} \circ i$ are Legendrian equivalent for any $C^{\prime} \in C\left(J^{1}\left(\mathbb{R}^{n}, \mathbb{R}\right), 0\right)$ satisfying $j^{l} C(0)=j^{l} C^{\prime}(0)$.

Lemma 5.5. (cf., [10, p.116 Lemma 7.2]) Let $\left\{L_{\sigma}\right\}_{\sigma \subset I_{r}}$ be a contact regular $r$-cubic configuration in $\left(J^{1}\left(\mathbb{R}^{n}, \mathbb{R}\right), 0\right)$ defined by $C \in C\left(J^{1}\left(\mathbb{R}^{n}, \mathbb{R}\right), 0\right)$. Then there exists $C^{\prime} \in C^{\theta}\left(J^{1}\left(\mathbb{R}^{n}, \mathbb{R}\right), 0\right)$ that also defines $\left\{L_{\sigma}\right\}_{\sigma \subset I_{r}}$.

By Lemma 5.5 we may consider the following other definition of finite determinacy of reticular Legendrian maps:

(1) The definition given by replacing $C\left(J^{1}\left(\mathbb{R}^{n}, \mathbb{R}\right), 0\right)$ to $C^{\theta}\left(J^{1}\left(\mathbb{R}^{n}, \mathbb{R}\right), 0\right)$.

(2) The definition given by replacing $C\left(J^{1}\left(\mathbb{R}^{n}, \mathbb{R}\right), 0\right)$ to $C_{Z}\left(J^{1}\left(\mathbb{R}^{n}, \mathbb{R}\right), 0\right)$.

(3) The definition given by replacing $C\left(J^{1}\left(\mathbb{R}^{n}, \mathbb{R}\right), 0\right)$ to $C_{Z}^{\theta}\left(J^{1}\left(\mathbb{R}^{n}, \mathbb{R}\right), 0\right)$.

Then the following holds:

Proposition 5.6. Let $\tilde{\pi} \circ i:(\tilde{\mathbb{L}}, 0) \rightarrow\left(J^{1}\left(\mathbb{R}^{n}, \mathbb{R}\right), 0\right) \rightarrow\left(\mathbb{R}^{n} \times \mathbb{R}, 0\right)$ be a reticular Legendrian map. Then

(A) If $\tilde{\pi} \circ i$ is l-determined of the original definition, then $\tilde{\pi} \circ i$ is $l$-determined of the definition (1).

(B) If $\tilde{\pi} \circ i$ is l-determined of the definition (1), then $\tilde{\pi} \circ i$ is l-determined of the definition (3).

(C) If $\tilde{\pi} \circ i$ is l-determined of the definition (3), then $\tilde{\pi} \circ i$ is $(l+1)$-determined of the definition (2).

(D) If $\tilde{\pi} \circ i$ is l-determined of the definition (2), then $\tilde{\pi} \circ i$ is l-determined of the original definition.

Proof. (A) Let $C \in C^{\theta}\left(J^{1}\left(\mathbb{R}^{n}, \mathbb{R}\right), 0\right)$ be an extension of $i$. Let $C^{\prime} \in$ $C^{\theta}\left(J^{1}\left(\mathbb{R}^{n}, \mathbb{R}\right), 0\right)$ satisfying $j^{l} C(0)=j^{l} C^{\prime}(0)$ be given. Since $C, C^{\prime} \in C\left(J^{1}\left(\mathbb{R}^{n}, \mathbb{R}\right), 0\right)$, 
we have that $\left.\tilde{\pi} \circ C^{\prime}\right|_{\tilde{\pi}}$ and $\tilde{\pi} \circ i$ are Legendrian equivalent.

(B) Let $C \in C_{Z}^{\theta}\left(J^{1}\left(\mathbb{R}^{n}, \mathbb{R}\right), 0\right)$ be an extension of $i$. Let $C^{\prime} \in C_{Z}^{\theta}\left(J^{1}\left(\mathbb{R}^{n}, \mathbb{R}\right), 0\right)$ satisfying $j^{l} C(0)=j^{l} C^{\prime}(0)$ be given. We define $C_{1}, C_{1}^{\prime} \in C^{\theta}\left(J^{1}\left(\mathbb{R}^{n}, \mathbb{R}\right), 0\right)$ by $C_{1}(Q, Z, P):=\left(q_{C}(Q, P), Z+z_{C}(Q, P), p_{C}(Q, P)\right), C_{1}^{\prime}(Q, Z, P):=\left(q_{C^{\prime}}(Q, P), Z+\right.$ $\left.z_{C^{\prime}}(Q, P), p_{C^{\prime}}(Q, P)\right)$. Then $C_{1}$ is an extension of $i$ and we have that $j^{l} C_{1}(0)=$ $j^{l} C_{1}^{\prime}(0)$. Therefore we have that $\left.\tilde{\pi} \circ C_{1}^{\prime}\right|_{\tilde{\mathbb{L}}}=\left.\tilde{\pi} \circ C^{\prime}\right|_{\tilde{\mathbb{L}}}$ and $\tilde{\pi} \circ i$ are Legendrian equivalent.

(D) Let $C \in C\left(J^{1}\left(\mathbb{R}^{n}, \mathbb{R}\right), 0\right)$ be an extension of $i$. Let $C^{\prime} \in C\left(J^{1}\left(\mathbb{R}^{n}, \mathbb{R}\right), 0\right)$ satisfying $j^{l} C(0)=j^{l} C^{\prime}(0)$ be given. We set $C_{1}:=\left.C\right|_{Z=0}, C_{1}^{\prime}:=\left.C^{\prime}\right|_{Z=0} \in C_{Z}\left(J^{1}\left(\mathbb{R}^{n}, \mathbb{R}\right), 0\right)$ and we have that $j^{l} C_{1}(0)=j^{l} C_{1}^{\prime}(0)$. Therefore $\left.\tilde{\pi} \circ C_{1}^{\prime}\right|_{\tilde{L}}=\left.\tilde{\pi} \circ C^{\prime}\right|_{\tilde{L}}$ and $\tilde{\pi} \circ i$ are Legendrian equivalent.

(C) Let $C \in C_{Z}\left(J^{1}\left(\mathbb{R}^{n}, \mathbb{R}\right), 0\right)$ be an extension of $i$. Let $C^{\prime} \in C_{Z}\left(J^{1}\left(\mathbb{R}^{n}, \mathbb{R}\right), 0\right)$ satisfying $j^{l+1} C(0)=j^{l+1} C^{\prime}(0)$ be given. Then there exist function germs $f(Q, P), g(Q, P) \in \mathcal{E}(2 n)$ such that $C^{*}(d z-p d q)=-f P d Q, C^{\prime *}(d z-p d q)=-g P d Q$. Indeed $f$ is defined by that $f P_{j}=-\frac{\partial z_{C}}{\partial Q_{j}}+p_{C} \frac{\partial q_{C}}{\partial Q_{j}}$ for $j=1, \ldots, n$. We define the diffeomorphism germs $\phi, \psi$ on $\left(J^{1}\left(\mathbb{R}^{n}, \mathbb{R}\right) \cap\{Z=0\}, 0\right)$ by $\phi(Q, P)=(Q, f P), \psi(Q, P)=$ $(Q, g P)$. We set $C_{1}:=C \circ \phi^{-1}, C_{1}^{\prime}:=C^{\prime} \circ \psi^{-1} \in C_{Z}^{\theta}\left(J^{1}\left(\mathbb{R}^{n}, \mathbb{R}\right), 0\right)$ Then $j^{l} \phi(0)$ and $j^{l} \psi(0)$ depend only on $j^{l+1} C(0)$, therefore we have that $j^{l} C_{1}(0)=j^{l} C_{1}^{\prime}(0)$. Since $\tilde{\pi} \circ i$ and $\left.\tilde{\pi} \circ C_{1}\right|_{\tilde{\mathbb{L}}}$ are Legendrian equivalent, it follows that $\left.\tilde{\pi} \circ C_{1}\right|_{\tilde{\mathbb{L}}}$ and $\left.\tilde{\pi} \circ C_{1}^{\prime}\right|_{\tilde{\mathbb{L}}}$ are Legendrian equivalent. Therefore we have that $\tilde{\pi} \circ i$ and $\left.\tilde{\pi} \circ C^{\prime}\right|_{\tilde{\mathbb{L}}}$ are Legendrian equivalent.

Theorem 5.7. Let $\tilde{\pi} \circ i:(\tilde{\mathbb{L}}, 0) \rightarrow\left(J^{1}\left(\mathbb{R}^{n}, \mathbb{R}\right), 0\right) \rightarrow\left(\mathbb{R}^{n} \times \mathbb{R}, 0\right)$ be a reticular Legendrian map. If $\tilde{\pi} \circ i$ is infinitesimally stable then $\tilde{\pi} \circ i$ is $(n+3)$-determined.

Proof. It is enough to prove $\tilde{\pi} \circ i$ is $(n+2)$-determined of Definition $5.4(3)$ and this is proved by an analogous method of Theorem 5.3. We write the sketch of the proof. Let $C \in C_{Z}^{\theta}\left(J^{1}\left(\mathbb{R}^{n}, \mathbb{R}\right), 0\right)$ be an extension of $i$. Then we may assume that $P_{C}$ has the form

$$
P_{C}=\left\{\left(Q,-\frac{\partial H}{\partial Q}(Q, p) ; H(Q, p)-\left\langle\frac{\partial H}{\partial Q}(Q, p), p\right\rangle,-\frac{\partial H}{\partial p}(Q, p), p\right)\right\}
$$

for some function germ $H(Q, p) \in \mathfrak{M}(2 n)^{2}$. Then $F(x, y, q, z)=H_{0}(x, y)+\langle y, q\rangle-z \in$ $\mathfrak{M}(r ; n+n+1)$ is a generating family of $\tilde{\pi} \circ i$, where $H_{0}(x, y)=H(x, 0, y) \in \mathfrak{M}(r ; n)^{2}$. Then $F$ is a reticular $\mathcal{P}$ - $\mathcal{K}$-infinitesimally stable unfolding of $H_{0}(x, y)$. It follows that $H_{0}$ is reticular $\mathcal{K}$ - $(n+2)$-determined. Let $C^{\prime} \in C_{Z}^{\theta}\left(J^{1}\left(\mathbb{R}^{n}, \mathbb{R}\right), 0\right)$ satisfying $j^{n+2} C(0)=j^{n+2} C^{\prime}(0)$ be given. There exists a function germ $H^{\prime}(Q, p) \in \mathfrak{M}(2 n)$ such that

$$
P_{C^{\prime}}=\left\{\left(Q,-\frac{\partial H^{\prime}}{\partial Q}(Q, p) ; H^{\prime}(Q, p)-\left\langle\frac{\partial H^{\prime}}{\partial Q}(Q, p), p\right\rangle,-\frac{\partial H^{\prime}}{\partial p}(Q, p), p\right)\right\} .
$$

Since $H=z-q p$ on $P_{C}$ and $H^{\prime}=z-q p$ on $P_{C^{\prime}}$, we have that $j^{n+2} H_{0}(0)=j^{n+2} H_{0}^{\prime}(0)$, where $H_{0}^{\prime}(x, y)=H^{\prime}(x, 0, y) \in \mathfrak{M}(r ; n)^{2}$. The function germ $G(x, y, q, z)=H_{0}^{\prime}(x, y)+$ $\langle y, q\rangle-z \in \mathfrak{M}(r ; n+n+1)$ is a generating family of $\left.\tilde{\pi} \circ C^{\prime}\right|_{\tilde{L}}$. Then there exist $\Phi(x, y) \in$ $\mathcal{B}(r ; n)$ and a unit $a \in \mathcal{E}(r ; n)$ such that $H_{0}=a \cdot H_{0}^{\prime} \circ \Phi$. We set $G^{\prime}(x, y, q, z)=$ $a(x, y) G(\Phi(x, y), q, z) \in \mathfrak{M}(r ; n+n+1)$. Then $G$ and $G^{\prime}$ are reticular $\mathcal{P}$ - $\mathcal{K}$-equivalent and $F$ and $G^{\prime}$ are reticular $\mathcal{P}$ - $\mathcal{K}$-infinitesimal stable unfoldings of $H_{0}(x, y)$. It follows that $F$ and $G$ are reticular $\mathcal{P}$ - $\mathcal{K}$-equivalent. Therefore $\tilde{\pi} \circ i$ and $\left.\tilde{\pi} \circ C^{\prime}\right|_{\tilde{\mathbb{L}}}$ are Legendrian equivalent. 
In order to prove Theorem 6.5 and Theorem 7.4, we require the following lemma:

LEMma 5.8. (cf., [3, p.53 Lemma 4.6]) Let $V, W$ be smooth manifolds with $Q$ a submanifold of $W, F$ be a topological space. We equip $C^{\infty}(V, W)$ with the Whitney $C^{\infty}$-topology. Let $j: F \rightarrow C^{\infty}(V, W)$ be a map (not necessary continuous). We suppose that: For each $f \in F$, there exist a manifold $E, e_{0} \in E$, and a continuous map $\phi: E \rightarrow F, \phi\left(e_{0}\right)=f$ such that the map $\Phi: E \times V \rightarrow W, \Phi(e, x)=j(\phi(e))(x)$, is smooth and transversal to $Q$. Then the set

$$
T=\{f \in F \mid j(f) \text { is transversal to } Q\}
$$

is dense in $F$.

6. Genericity of reticular Lagrangian maps. Let $J^{l}(2 n, 2 n)$ be the set of $l$ jets of map germs from $\left(T^{*} \mathbb{R}^{n}, 0\right)$ to $\left(T^{*} \mathbb{R}^{n}, 0\right)$ and $S^{l}(n)$ be the Lie group in $J^{l}(2 n, 2 n)$ consists of $l$-jets of symplectic diffeomorphism germs on $\left(T^{*} \mathbb{R}^{n}, 0\right)$.

We consider the Lie group $L^{l}(2 n) \times L^{l}(2 n)$ acts on $J^{l}(2 n, 2 n)$ as coordinate changes of the source and target spaces. We also consider the Lie subgroup $r L a^{l}(n)$ of $L^{l}(2 n) \times$ $L^{l}(2 n)$ consists of $l$-jets of reticular diffeomorphisms on the source space and $l$-jets of Lagrangian equivalences of $\pi$ on the target space:

$$
\begin{array}{r}
r L a^{l}(n)=\left\{\left(j^{l} \phi(0), j^{l} \Theta(0)\right) \in L^{l}(2 n) \times L^{l}(2 n) \mid \phi\right. \text { is a reticular diffeomorphism on } \\
\left.\left(T^{*} \mathbb{R}^{n}, 0\right), \Theta \text { is a Lagrangian equivalence of } \pi\right\} .
\end{array}
$$

The group $r L a^{l}(n)$ acts on $J^{l}(2 n, 2 n)$ and $S^{l}(n)$ is invariant under this action. Let $S$ be a symplectic diffeomorphism germ on $\left(T^{*} \mathbb{R}^{n}, 0\right)$ and set $z=j^{l} S(0)$. We denote the orbit $r L a^{l}(n) \cdot z$ by $[z]$. Then

$$
[z]=\left\{j^{l} S^{\prime}(0) \in S^{l}(n) \mid \pi \circ i \text { and }\left.\pi \circ S^{\prime}\right|_{\mathbb{L}} \text { are Lagrangian equivalent }\right\} .
$$

In this section we denote by $X_{f}$ the Hamiltonian vector field on $\left(T^{*} \mathbb{R}^{n}, 0\right)$ for a function germ $f$ on $\left(T^{*} \mathbb{R}^{n}, 0\right)$. That is

$$
X_{f}=\sum_{j=1}^{n}\left(\frac{\partial f}{\partial p_{j}} \frac{\partial}{\partial q_{j}}-\frac{\partial f}{\partial q_{j}} \frac{\partial}{\partial p_{j}}\right) .
$$

We denote by $V I_{S}$ the vector space consists of infinitesimal symplectic transformations of $S$ and denote by $V I_{S}^{0}$ the subspace of $V I_{S}$ consists of germs which vanishes on 0 . We denote by $V L_{T * \mathbb{R}^{n}}$ by the vector space consists of infinitesimal Lagrangian equivalences of $\pi$ and denote by $V L_{T^{*} \mathbb{R}^{n}}^{0}$ by the subspace of $V L_{T^{*} \mathbb{R}^{n}}$ consists of germs which vanishes at 0 .

We denote by $V_{\mathbb{L}}^{0}$ the vector space consists of infinitesimal reticular diffeomorphisms on $\left(T^{*} \mathbb{R}^{n}, 0\right)$ which vanishes at 0 :

$$
V_{\mathbb{L}}^{0}=\left\{\xi \in X\left(T^{*} \mathbb{R}^{n}, 0\right) \mid \xi \text { is tangent to } L_{\sigma}^{0} \text { for all } \sigma \subset I_{r}, \xi(0)=0\right\} .
$$

From now on, we denote by $\mathcal{E}_{T^{*} \mathbb{R}^{n}}$ the ring of smooth function germs on $\left(T^{*} \mathbb{R}^{n}, 0\right)$ and denote by $\mathfrak{M}_{T^{*} \mathbb{R}^{n}}$ its maximal ideal. We also denote other notations analogously. 
Lemma 6.1. (1) A vector field germ $v$ on $\left(T^{*} \mathbb{R}^{n}, 0\right)$ along $S$ belongs to $V I_{S}$ if and only if there exists a function germ $f$ on $\left(T^{*} \mathbb{R}^{n}, 0\right)$ such that $v=X_{f} \circ S$,

(2) A vector field germ $\eta$ on $\left(T^{*} \mathbb{R}^{n}, 0\right)$ belongs to $V L_{T^{*} \mathbb{R}^{n}}$ if and only if there exists a fiber preserving function germ $H$ on $\left(T^{*} \mathbb{R}^{n}, 0\right)$ such that $\eta=X_{H}$.

(3) A vector field germ $\xi$ on $\left(T^{*} \mathbb{R}^{n}, 0\right)$ belongs to $V_{\mathbb{L}}^{0}$ if and only if there exists a function germ $g \in B_{0}$ such that $\xi=X_{g}$, where $B_{0}=\left\langle q_{1} p_{1}, \ldots, q_{r} p_{r}\right\rangle_{\mathcal{E}_{T^{*} \mathbb{R}^{n}}}+$ $\mathfrak{M}_{T^{*} \mathbb{R}^{n}}\left\langle q_{r+1}, \ldots, q_{n}\right\rangle$ is a submodule of $\mathcal{E}_{T^{*} \mathbb{R}^{n}}$.

By this lemma we have that:

$$
V I_{S}^{0}=\left\{v:\left(T^{*} \mathbb{R}^{n}, 0\right) \rightarrow\left(T\left(T^{*} \mathbb{R}^{n}\right), 0\right) \mid v=X_{f} \circ S \text { for some } f \in \mathfrak{M}_{T^{*} \mathbb{R}^{n}}^{2},\right.
$$

$V L_{T^{*} \mathbb{R}^{n}}^{0}$

$=\left\{\eta \in X\left(T^{*} \mathbb{R}^{n}, 0\right) \mid \eta=X_{H}\right.$ for some fiber preserving function germ $\left.H \in \mathfrak{M}_{T^{*} \mathbb{R}^{n}}^{2}\right\}$,

$$
V_{\mathbb{L}}^{0}=\left\{\xi \in X\left(T^{*} \mathbb{R}^{n}, 0\right) \mid \xi=X_{g} \text { for some } g \in B_{0}\right\}
$$

We define the homomorphism $t S: V_{\mathbb{L}}^{0} \rightarrow V I_{S}^{0}$ by $t S(v)=S_{*} v$ and define the homomorphism $w S: V L_{T^{*} \mathbb{R}^{n}}^{0} \rightarrow V I_{S}^{0}$ by $w S(\eta)=\eta \circ S$.

Lemma 6.2. Let $S$ be a symplectic diffeomorphism germ on $\left(T^{*} \mathbb{R}^{n}, 0\right)$ and set $z=j^{l} S(0)$. Then

$$
T_{z}\left(r L a^{l}(n) \cdot z\right)=\pi_{l}\left(t S\left(V_{\mathbb{L}}^{0}\right)+w S\left(V L_{T^{*} \mathbb{R}^{n}}^{0}\right)\right) .
$$

We denote $V I_{S}^{l}$ the subspace of $V I_{S}$ consists of infinitesimal symplectic transformation germs of $S$ whose $l$-jets are 0 :

$$
V I_{S}^{l}=\left\{v \in V I_{S} \mid j^{l} v(0)=0\right\} .
$$

We consider the surjective projection $\pi_{l}: V I_{S} \rightarrow T_{z}\left(S^{l}(n)\right)$. Since $\left(j^{l} S\right)_{*}\left(\frac{\partial}{\partial q_{j}}\right)=$ $\pi_{l}\left(S_{*} \frac{\partial}{\partial q_{j}}\right),\left(j^{l} S\right)_{*}\left(\frac{\partial}{\partial p_{j}}\right)=\pi_{l}\left(S_{*} \frac{\partial}{\partial p_{j}}\right)$, it follows that $j^{l} S$ is transversal to $[z]$ if and only if $\left(j^{l} S\right)_{*}\left(T_{0}\left(T^{*} \mathbb{R}^{n}\right)\right)+T_{z}[z]=T_{z}\left(S^{l}(n)\right)$ and this holds if and only if

$$
\left(\pi_{l}\right)^{-1}\left(\left(j^{l} S\right)_{*}\left(T_{0}\left(T^{*} \mathbb{R}^{n}\right)\right)+t S\left(V_{\mathbb{L}}^{0}\right)+w S\left(V L_{T^{*} \mathbb{R}^{n}}^{0}\right)\right)+V I_{S}^{l+1}=V I_{S}
$$

and this holds if and only if

$$
\left.t S\left(V_{\mathbb{L}}^{0}\right)+w S\left(V L_{T^{*} \mathbb{R}^{n}}\right)\right)+V I_{S}^{l+1}=V I_{S} .
$$

Let $N, M$ be $2 n$ dimensional symplectic manifolds. We denote $S(N, M)$ the space of symplectic embeddings from $N$ to $M$ with the topology induced by the Whitney $C^{\infty}$-topology of $C^{\infty}(N, M)$. We define that

$J_{S}^{l}(N, M)=\left\{j^{l} S\left(u_{0}\right) \in J^{l}(N, M) \mid\right.$

$S:\left(N, u_{0}\right) \rightarrow M$ is a symplectic embedding germ, $\left.u_{0} \in N\right\}$.

Proposition 6.3. $S(N, M)$ is a Baire space. 
This is proved by an analogous method of the assertion that $C^{\infty}(N, M)$ is a Baire space (cf., [3, p.44 Proposition 3.3]).

The following theorem is the special case of [4, p.94 Theorem 0.1]. We consider the graph maps $N \rightarrow N \times M$ of symplectic diffeomorphisms $N \rightarrow M$ instead of isotropic maps $X \rightarrow M$ in [4]. We give the self-contained proof.

Theorem 6.4. Let $N, M$ be $2 n$ dimensional symplectic manifolds. Let $Q_{j}, j=$ $1,2, \ldots$ be submanifolds of $J_{S}^{l}(N, M)$. Then the set

$$
T=\left\{S \in S(N, M) \mid j^{l} S \text { is transversal to } Q_{j} \text { for all } j \in \mathbb{N}\right\}
$$

is residual set in $S(N, M)$. In particular $T$ is dense.

Proof. We apply for Lemma 5.8 that $V=N, W=J_{S}^{l}(N, M)$, and $F=$ $S(N, M)$. We reduce our assertion to local situations by choosing a countable covering of $Q_{j}$ by sufficiently small compact sets $K_{j, k}$ 's. Then the sets $T_{j, k}=\{S \in$ $S(N, M) \mid j^{l} S$ is transversal to $Q_{j}$ at $\left.K_{j, k}\right\}$ are open set by Lemma 5.8 and we have that $T=\cap T_{j, k}$ We fix a symplectic embedding $S \in S(N, M)$. For each $u_{0} \in N$ there exist local symplectic coordinate systems of $N$ around $u_{0}$ and $M$ around $S\left(u_{0}\right)$ such that $S$ is given by $(q, p) \mapsto(q, p)$ around 0 .

For each $j, k$ we take $E$ by a sufficiently small neighborhood of 0 in $P(2 n, 1 ; l+1)$ and take a smooth function $\rho: T^{*} \mathbb{R}^{n} \rightarrow[0,1]$ such that $\rho$ is 1 on a neighborhood of 0 and zero outside a compact set, where $P(2 n, 1 ; l+1)$ is the set of not higher than $(l+1)$-degree polynomials on $2 n$ variables.

For each $H \in E$ we define $H^{\prime}(Q, p)=\rho(Q, p) H(Q, p)-\langle Q, p\rangle$ and $\psi_{H^{\prime}}(Q, p)=(Q$, $\left.-\frac{\partial H^{\prime}}{\partial Q}(Q, p)\right)$ for $(Q, p) \in T^{*} \mathbb{R}^{n}$ around 0 . Then there exists a neighborhood $U$ of 0 in $T^{*} \mathbb{R}^{n}$ such that $\psi_{H^{\prime}}$ is a embedding on $U$ and equal to the identity map outside a compact set for any $H \in E$. Therefore there exists a neighborhood $U^{\prime}$ of 0 in $T^{*} \mathbb{R}^{n}$ such that the map $E \rightarrow C^{\infty}\left(U^{\prime}, U\right),\left.H \mapsto\left(\psi_{H}^{-1}\right)\right|_{U^{\prime}}$ is well defined and continuous. Each $\left.\left(\psi_{H}^{-1}\right)\right|_{U^{\prime}}$ is equal the identity map outside a compact set around 0 . We set that

$$
\phi(H)(Q, P)=\left(-\frac{\partial H^{\prime}}{\partial p}(Q, p), p\right) \circ\left(\psi_{H^{\prime}}\right)^{-1}(Q, P) \text { for }(Q, P) \in U^{\prime} .
$$

Then $\phi(H)$ is a symplectic diffeomorphism around $u_{0}$ which has the canonical relation with the generating function $H^{\prime}(Q, p)$ and equal to $S$ outside a compact set. It follows that the source space of $\phi(H)$ may be extended naturally to $E \times N$. We also denote this by $\phi_{H} \in S(N, M)$. Then the map

$$
\Phi: E \times N \rightarrow J_{S}^{l}(N, M), \Phi(H, q, p)=j^{l}\left(\phi_{H}\right)(q, p)
$$

is a submersion around $\left(0, u_{0}\right)$ Therefore $\Phi$ is transversal to $K_{j, k}$. So we have the result.

For $\tilde{S} \in S\left(U, T^{*} \mathbb{R}^{n}\right)$ we define the continuous map $j_{0}^{l} \tilde{S}: U \rightarrow S^{l}(n)$ by $w$ to the $l$-jet of $\tilde{S}_{w}$ at 0 . We remark that $j^{l} \tilde{S}_{w}(0)$ has the form $j^{l} \tilde{S}_{w}(0)=\left(w, \tilde{S}(w), j_{0}^{l} \tilde{S}_{w}(0)\right)$.

TheOREM 6.5. Let $U$ be a neighborhood of 0 in $T^{*} \mathbb{R}^{n}$, and let $Q_{1}, Q_{2}, \ldots$ are submanifolds of $S^{l}(n)$. Then the set $L_{U}=\left\{(q, p) \in U \mid q=p_{1}=\cdots=p_{r}=0\right\}$ and

$$
T=\left\{\tilde{S} \in S\left(U, T^{*} \mathbb{R}^{n}\right) \mid j_{0}^{l} \tilde{S} \text { is transversal to } Q_{j} \text { on } L_{U} \text { for all } j\right\}
$$

is a residual set in $S\left(U, T^{*} \mathbb{R}^{n}\right)$. 
Proof. We set $Q_{j}^{\prime}=L_{U} \times T^{*} \mathbb{R}^{n} \times Q_{j} \subset J_{S}^{l}\left(U, T^{*} \mathbb{R}^{n}\right)$. We choose a countable covering of $Q_{j}^{\prime}$ by sufficiently small compact sets $K_{j, k}^{\prime} s$ for all $j$. We apply $N=$ $V, M=T^{*} \mathbb{R}^{n}$ for Theorem 6.4. We have that

$$
T=\left\{\tilde{S} \in S\left(U, T^{*} \mathbb{R}^{n}\right) \mid j^{l} \tilde{S} \text { is transversal to } Q_{j}^{\prime} \text { on } K_{j, k} \text { for all } j, k\right\}
$$

It follows that $T$ is a residual set in $S\left(U, T^{*} \mathbb{R}^{n}\right)$.

We have the following theorem by using Ishikawa's method (cf., [5, Section 5]) for our situation.

TheOREM 6.6. Let $\pi \circ i:(\mathbb{L}, 0) \rightarrow\left(T^{*} \mathbb{R}^{n}, 0\right) \rightarrow\left(\mathbb{R}^{n}, 0\right)$ be a reticular Lagrangian map. Let $S$ be an extension of $i$ and $l \geq n+1$. Let $B=$ $\left\langle q_{1} p_{1}, \ldots, q_{r} p_{r}, q_{r+1}, \ldots, q_{n}\right\rangle_{\mathcal{E}_{T^{*} \mathbb{R}^{n}}}$ be a submodule of $\mathcal{E}_{T^{*} \mathbb{R}^{n}}$. Then the following are all equivalent:

(s) $\pi \circ i$ is stable.

(t) $j_{0}^{l} S$ is transversal to $\left[j_{0}^{l} S(0)\right]$ at 0 .

(a') $\mathcal{E}_{T^{*} \mathbb{R}^{n}} /\left(B+\mathfrak{M}_{T^{*} \mathbb{R}^{n}}^{l+2}\right)$ is generated by $1, p_{1} \circ S, \ldots, p_{n} \circ S$ as an $\mathcal{E}_{q^{-m o d u l e}}$ via $(\pi \circ S)^{*}$.

(a") $\mathcal{E}_{T^{*} \mathbb{R}^{n}} / B$ is generated by $1, p_{1} \circ S, \ldots, p_{n} \circ S$ as an $\mathcal{E}_{q}$-module via $(\pi \circ S)^{*}$.

(is) $\pi \circ i$ is infinitesimally stable.

Proof. $(\mathrm{s}) \Rightarrow(\mathrm{t})$. Let $\tilde{S} \in S\left(U, T^{*} \mathbb{R}^{n}\right)$ be a representative of $S$. By theorem 6.5 there exists a symplectic embedding $\tilde{S}^{\prime}$ around $\tilde{S}$ such that $j_{0}^{l} \tilde{S}^{\prime}$ is transversal to $\left[j_{0}^{l} S(0)\right]$ at $w=\left(0, \ldots, 0, p_{r+1}, \ldots, p_{n}\right) \in U$. Since $\pi \circ i$ is stable, $\pi \circ i$ and $\left.\pi \circ \tilde{S_{w}^{\prime}}\right|_{\mathbb{L}}$ are Lagrangian equivalent. This means that $\left[j_{0}^{l} \tilde{S_{w}^{\prime}}(0)\right]=\left[j_{0}^{l} S(0)\right]$ and hence $j_{0}^{l} S$ is transversal to $\left[j_{0}^{l} S(0)\right]$ at 0 .

$(\mathrm{t}) \Leftrightarrow\left(\mathrm{a}^{\prime}\right)$. By Lemma 6.1, we have that the condition $(\mathrm{t})$ is equivalent to the condition: For any function germ $f$ on $\left(T^{*} \mathbb{R}^{n}, 0\right)$, there exist a fiber preserving function germ $H$ on $\left(T^{*} \mathbb{R}^{n}, 0\right)$ and a function germ $g \in B$ such that $\pi_{l}\left(X_{f} \circ S\right)=\pi_{l}\left(S_{*} X_{g}+X_{H} \circ S\right)$. This is equivalent to the condition: For any function germ $f$ on $\left(T^{*} \mathbb{R}^{n}, 0\right)$, there exist a fiber preserving function germ $H$ on $\left(T^{*} \mathbb{R}^{n}, 0\right)$ and a function germ $g \in B$ such that $f \circ S-g-H \circ S \in \mathfrak{M}_{T^{*} \mathbb{R}^{n}}^{l+2}$. This is equivalent to the condition: For any function germ $f$ on $\left(T^{*} \mathbb{R}^{n}, 0\right)$, there exist a fiber preserving function germ $H$ on $\left(T^{*} \mathbb{R}^{n}, 0\right)$ such that $f-H \circ S \in B+\mathfrak{M}_{T * \mathbb{R}^{n}}^{l+2}$. This is equivalent to (a').

$\left(\mathrm{a}^{\prime}\right) \Leftrightarrow\left(\mathrm{a}^{\prime \prime}\right)$. We need only to prove $\left(\mathrm{a}^{\prime}\right) \Rightarrow\left(\mathrm{a}^{\prime \prime}\right)$. By Margrange preparation theorem, the condition (a') is equivalent that $\mathcal{E}_{T^{*} \mathbb{R}^{n}} /\left((\pi \circ S)^{*} \mathfrak{M}_{\mathbb{R}^{n}} \mathcal{E}_{T^{*} \mathbb{R}^{n}}+B+\mathfrak{M}_{T^{*} \mathbb{R}^{n}}^{l+2}\right)$ is generated by $1, p_{1} \circ S, \ldots, p_{n} \circ S$ over $\mathbb{R}$. This means that

$$
\mathfrak{M}_{T^{*} \mathbb{R}^{n}}^{n+1} \subset(\pi \circ S)^{*} \mathfrak{M}_{\mathbb{R}^{n}} \mathcal{E}_{T^{*} \mathbb{R}^{n}}+B+\mathfrak{M}_{T^{*} \mathbb{R}^{n}}^{l+2}
$$

Since $\mathfrak{M}_{T^{*} \mathbb{R}^{n}}^{l+2} \subset \mathfrak{M}_{T^{*} \mathbb{R}^{n}}^{n+2}$, it follows that

$$
\mathfrak{M}_{T^{*} \mathbb{R}^{n}}^{n+1} \subset(\pi \circ S)^{*} \mathfrak{M}_{\mathbb{R}^{n}} \mathcal{E}_{T^{*} \mathbb{R}^{n}}+B+\mathfrak{M}_{T^{*} \mathbb{R}^{n}}^{n+2}
$$

Therefore we have that

$$
\mathfrak{M}_{T^{*} \mathbb{R}^{n}}^{n+1} \subset(\pi \circ S)^{*} \mathfrak{M}_{\mathbb{R}^{n}} \mathcal{E}_{T^{*} \mathbb{R}^{n}}+B
$$

This means (a").

$(\mathrm{a} ") \Leftrightarrow($ is). The condition (a") is equivalent to the condition (a) in Theorem 4.1 and this is equivalent to (is). 
(t) \&(is) $\Rightarrow(\mathrm{s})$. Since $j_{0}^{l} S$ is transversal to $\left[j_{0}^{l} S(0)\right]$, it follows that there exist a representative $\tilde{S} \in S\left(U, T^{*} \mathbb{R}^{n}\right)$ of $S$ and a neighborhood $W_{\tilde{S}}$ of $\tilde{S}$ such that for any $\tilde{S}^{\prime} \in W_{\tilde{S}}$ there exists $w \in U$ such that $j_{0}^{l} \tilde{S}^{\prime}$ is transversal to $\left[j_{0}^{l} S(0)\right]$ at $w$. Since $j_{0}^{l} \tilde{S_{w}^{\prime}}(0) \in\left[j_{0}^{l} S(0)\right]$, it follows that there exists a symplectic embedding germ $S_{\tilde{S}}^{\prime \prime}$ on $\left(T^{*} \mathbb{R}^{n}, 0\right)$ such that $\pi \circ i$ and $\left.\pi \circ S^{\prime \prime}\right|_{\mathbb{L}}$ is Lagrangian equivalent and $j_{0}^{l} S^{\prime \prime}(0)=j_{0}^{l} \tilde{S_{w}^{\prime}}(0)$. Since $\pi \circ i$ is infinitesimally stable, it follows that $\pi \circ i$ is $l$-determined by Theorem 5.3. Therefore we have that $\left.\pi \circ S^{\prime \prime}\right|_{\mathbb{L}}$ is also $l$-determined. It follows that $\left.\pi \circ S^{\prime \prime}\right|_{\mathbb{L}}$ and $\left.\pi \circ \tilde{S_{w}^{\prime}}\right|_{\mathbb{L}}$ is Lagrangian equivalent. This means that $\pi \circ i$ is stable. $\square$

Let $S$ be a symplectic diffeomorphism germ on $\left(T^{*} \mathbb{R}^{n}, 0\right), F(x, y, q) \in \mathfrak{M}(r ; k+$ $n)^{2}$, and $f(x, y) \in \mathfrak{M}(r ; k)^{2}$. We denote $[S],[F],[f]$ by the equivalence classes of $S, F, f$ under the Lagrangian equivalence, the stable reticular $\mathcal{P}$ - $\mathcal{R}^{+}$-equivalence, and the stable reticular $\mathcal{R}$-equivalence respectively. Then the following holds:

Lemma 6.7. Let $\pi \circ i_{j}:(\mathbb{L}, 0) \rightarrow\left(T^{*} \mathbb{R}^{n}, 0\right) \rightarrow\left(\mathbb{R}^{n}, 0\right)$ be stable reticular Lagrangian maps with generating families $F_{j}(x, y, q) \in \mathfrak{M}\left(r ; k_{j}+n\right)^{2}$, and $S_{j}$ be extensions of $i_{j}$ for $j=1,2$. Then $\left[S_{1}\right]=\left[S_{2}\right]$ if and only if $\left[F_{1}\right]=\left[F_{2}\right]$ and this holds if and only if $\left[\left.F_{1}\right|_{q=0}\right]=\left[\left.F_{2}\right|_{q=0}\right]$.

Proof. $\left[S_{1}\right]=\left[S_{2}\right]$ if and only if reticular Lagrangian maps $\pi \circ i_{1}$ and $\pi \circ i_{2}$ are Lagrangian equivalent by Lemma 5.2 , and this holds if and only if $\left[F_{1}\right]=\left[F_{2}\right]$ by $\left[9\right.$, p.577, Theorem 3.2(3)], and if this holds then $\left[\left.F_{1}\right|_{q=0}\right]=\left[\left.F_{2}\right|_{q=0}\right]$. Conversely suppose that $\left[\left.F_{1}\right|_{q=0}\right]=\left[\left.F_{2}\right|_{q=0}\right]$. Since $F_{1}$ and $F_{2}$ are reticular $\mathcal{P}$ - $\mathcal{R}^{+}$-stable unfoldings of $\left[\left.F_{1}\right|_{q=0}\right]$ and $\left[\left.F_{2}\right|_{q=0}\right]$ respectively and $\left.F_{1}\right|_{q=0}$ and $\left.F_{2}\right|_{q=0}$ are stably reticular $\mathcal{R}$-equivalent, it follows that $\left[F_{1}\right]=\left[F_{2}\right]$.

Corollary 6.8. Let $\pi \circ i:(\mathbb{L}, 0) \rightarrow\left(T^{*} \mathbb{R}^{n}, 0\right) \rightarrow\left(\mathbb{R}^{n}, 0\right)$ be a stable reticular Lagrangian map. Then there exist a neighborhood $U$ of 0 in $T^{*} \mathbb{R}^{n}$ and $\tilde{S} \in S\left(U, T^{*} \mathbb{R}^{n}\right)$ with $i=\left.\tilde{S}_{0}\right|_{\mathbb{L}}$ ( that is, $\tilde{S}$ is a representative of an extension of $\left.i\right)$ such that reticular Lagrangian maps $\left.\pi \circ \tilde{S_{w}}\right|_{\mathbb{L}}$ are stable for all $w \in U$

Proof. By Theorem 6.6 (a'), the stability of reticular Lagrangian maps are determined by the $(n+1)$-jets of $\pi \circ \tilde{S_{w}}$ for $w \in U$. Therefore we have the result by shrinking $U$ if necessary.

Let $\pi \circ i:(\mathbb{L}, 0) \rightarrow\left(T^{*} \mathbb{R}^{n}, 0\right) \rightarrow\left(\mathbb{R}^{n}, 0\right)$ be a stable reticular Lagrangian map. We say that $\pi \circ i$ is simple if there exist a neighborhood $U$ of 0 in $T^{*} \mathbb{R}^{n}$ and $\tilde{S} \in S\left(U, T^{*} \mathbb{R}^{n}\right)$ such that $i=\left.\tilde{S}_{0}\right|_{\mathbb{L}}$ and $\left\{\tilde{S}_{w} \mid w \in U\right\}$ is covered by finite orbits $\left[S_{1}\right], \ldots,\left[S_{m}\right]$ for symplectic diffeomorphism germs $S_{1}, \ldots, S_{m}$ on $\left(T^{*} \mathbb{R}^{n}, 0\right)$.

Lemma 6.9. Let $\pi \circ i:(\mathbb{L}, 0) \rightarrow\left(T^{*} \mathbb{R}^{n}, 0\right) \rightarrow\left(\mathbb{R}^{n}, 0\right)$ be a stable reticular Lagrangian map. Then $\pi \circ i$ is simple if and only if there exist a neighborhood $U_{z}$ of $z=j_{0}^{n+1} S(0)$ in $S^{n+1}(n)$ and $z_{1}, \ldots, z_{m} \in S^{n+1}(n)$ such that $U_{z} \subset\left[z_{1}\right] \cup \cdots \cup\left[z_{m}\right]$.

Proof. Suppose that $\pi \circ i$ is simple. Then there exists a representative $\tilde{S}: U \rightarrow$ $T^{*} \mathbb{R}^{n}$ of an extension of $i$ and symplectic diffeomorphism germs $\left[S_{1}\right], \ldots,\left[S_{m}\right]$ on $\left(T^{*} \mathbb{R}^{n}, 0\right)$ such that

$$
\left\{\tilde{S}_{w} \mid w \in U\right\} \subset\left[S_{1}\right] \cup \cdots \cup\left[S_{m}\right] .
$$

Since $\pi \circ i$ is stable, it follows that $j_{0}^{n+1} \tilde{S}$ is transversal to $[z]$ at 0 by Theorem 3.1. This means that there exists a neighborhood $U_{z}$ of $z$ in $S^{l}(n)$ such that $U_{z} \subset$ $\cup_{w \in U}\left[j_{0}^{n+1} \tilde{S}(w)\right]$. It follows that $U_{z} \subset\left[j^{n+1} S_{1}(0)\right] \cup \cdots \cup\left[j^{n+1} S_{m}(0)\right]$. 
Conversely suppose that there exist a neighborhood $U_{z}$ of $z$ in $S^{n+1}(n)$ and $z_{1}, \ldots, z_{m} \in S^{n+1}(n)$ such that $U_{z} \subset\left[z_{1}\right] \cup \cdots \cup\left[z_{m}\right]$. Since the map $j_{0}^{n+1} \tilde{S}: U \rightarrow$ $S^{n+1}(n)$ is continuous, there exists a neighborhood $U^{\prime}$ of 0 in $U$ such that $j_{0}^{n+1} \tilde{S}(w) \in$ $U_{z}$ for any $w \in U^{\prime}$. Then we have that $\cup_{w \in U^{\prime}} j_{0}^{n+1} \tilde{S}(w) \subset\left[z_{1}\right] \cup \cdots \cup\left[z_{m}\right]$. Choose symplectic diffeomorphism germs $S_{1}, \ldots, S_{m}$ on $\left(T^{*} \mathbb{R}^{n}, 0\right)$ such that $j^{n+1} S_{j}(0)=z_{j}$ for $j=1, \ldots, m$. By Corollary 6.8 , we may assume that each $\left.\pi \circ S_{j}\right|_{\mathbb{L}}$ is stable, therefore $(n+1)$-determined. For any $w \in U^{\prime}$ we have that there exists $j \in\{1, \ldots, m\}$ such that $j_{0}^{n+1} \tilde{S}_{w}(0) \in\left[j^{n+1} S_{j}(0)\right]$. It follows that reticular Lagrangian maps $\left.\pi \circ \tilde{S_{w}}\right|_{\mathbb{L}}$ and $\left.\pi \circ S_{j}\right|_{\mathbb{L}}$ are Lagrangian equivalent. Therefore $\tilde{S_{w}} \in\left[S_{j}\right]$. We have (1).

Lemma 6.10. A stable reticular Lagrangian map $\pi \circ i$ is simple if and only if the function germ $F(x, y, 0) \in \mathfrak{M}(r ; k)^{2}$ is $\mathcal{R}$-simple singularity for a generating family $F(x, y, q) \in \mathfrak{M}(r ; k+n)^{2}$ of $\pi \circ i$.

Proof. Let $S$ be an extension of $i$ and $\tilde{S} \in S\left(U, T^{*} \mathbb{R}^{n}\right)$ be a representative of $S$.

Suppose that $F(x, y, 0)$ is $\mathcal{R}$-simple. The simplicity of reticular Lagrangian maps is invariant under Lagrangian equivalences, we may assume that the map germ $(Q, P) \mapsto(Q, p \circ S(Q, P))$ is a diffeomorphism germ on $\left(\mathbb{R}^{2 n}, 0\right)$. We consider symplectic diffeomorphism germs $\tilde{S}_{w}$ on $\left(\mathbb{R}^{2 n}, 0\right)$ for $w \in U$ near 0 . Then there exist function germs $H_{w}(Q, p) \in \mathfrak{M}(2 n)^{2}$ such that the canonical relation $P_{w}$ associated with $\tilde{S}_{w}$ has the form:

$$
P_{w}=\left\{\left(Q,-\frac{\partial H_{w}}{\partial Q}(Q, p),-\frac{\partial H_{w}}{\partial p}(Q, p), p\right) \in\left(T^{*} \mathbb{R}^{n} \times T^{*} \mathbb{R}^{n},(0,0)\right)\right\} .
$$

Then the function germ $F_{w}(x, y, q)=H_{w}^{\prime}(x, y)+\langle y, q\rangle \in \mathfrak{M}(r ; n+n)^{2}$ is a generating family of $\left.\pi \circ \tilde{S}_{w}\right|_{\mathbb{L}}$, where $H_{w}^{\prime} \in \mathfrak{M}(r ; n)^{2}$ is defined by $H_{w}^{\prime}(x, y)=H_{w}(x, 0, y)$. Since $F_{0}$ is a generating family of $\pi \circ i$, we have that $F_{0}(x, y, 0)\left(=H_{0}^{\prime}(x, y)\right)$ is stably $\mathcal{R}$ equivalent to $F(x, y, 0)$. Therefore we have that $H_{0}^{\prime}$ is $\mathcal{R}$-simple. Then there exists $f_{1}, \ldots, f_{m} \in \mathfrak{M}(r ; n)$ and a neighborhood $V$ of $j^{n+2} H_{0}^{\prime}(0)$ in $J^{n+2}(r+n, 1)$ such that $V \subset\left[j^{n+2} f_{1}(0)\right] \cup \cdots \cup\left[j^{n+2} f_{m}(0)\right]$.

Since the $(n+2)$-jet of $H_{S}^{\prime}$ is determined by the $(n+1)$-jet of $S$, there exists a neighborhood $U^{\prime}$ of 0 in $U$ such that the map germ

$$
U^{\prime} \rightarrow J^{n+2}(r+n, 1), w \mapsto j^{n+2} H_{w}^{\prime}(0)
$$

is well defined and continuous.

Let $U^{\prime \prime}$ be the inverse image of $V$ by the above map. Then for any $w \in U^{\prime \prime}$ the reticular Lagrangian map $\left.\pi \circ \widetilde{S}_{w}\right|_{\mathbb{L}}$ has a generating family which is reticular $\mathcal{P}-\mathcal{R}^{+}$equivalent to $f_{j}(x, y)+\langle y, q\rangle \in \mathfrak{M}(r ; n+n)^{2}$ for some $j$ because $\left.\pi \circ \tilde{S}_{w}\right|_{\mathbb{L}}$ is stable by Corollary 6.8 and hence $(n+1)$-determined. It follow that $\left\{\tilde{S}_{w} \mid w \in U^{\prime \prime}\right\} \subset\left[S_{1}\right] \cup \cdots \cup$ $\left[S_{m}\right]$, where $S_{j}$ is an extension of a reticular Lagrangian embedding which defines a reticular Lagrangian map with the generating family $f_{j}(x, y)+\langle y, q\rangle \in \mathfrak{M}(r ; n+n)^{2}$. This means that $\pi \circ i$ is simple.

Conversely suppose that $\pi \circ i$ is simple. Let $S_{0}$ be an extension of $i$. we may assume that there exists a function germ $H_{0}(Q, p) \in \mathfrak{M}(2 n)^{2}$ such that the canonical relation $P_{S_{0}}$ associated with $S_{0}$ has the form:

$$
P_{S_{0}}=\left\{\left(Q,-\frac{\partial H_{0}}{\partial Q}(Q, p),-\frac{\partial H_{0}}{\partial p}(Q, p), p\right) \in\left(T^{*} \mathbb{R}^{n} \times T^{*} \mathbb{R}^{n},(0,0)\right)\right\} .
$$

This means that for a function germ $H$ around $H_{0}$ there exists a symplectic diffeomorphism germ $S_{H}$ on $\left(T^{*} \mathbb{R}^{n}, 0\right)$ with the canonical relation $P_{S_{H}}$ which has the 
form same as $P_{S_{0}}$. Then the $(n+1)$-jet of $S_{H}$ is determined by the $(n+2)$-jet of $H$. The function germ $H_{0}^{\prime}(x, 0, y)+\langle y, q\rangle \in \mathfrak{M}(r ; n+n)^{2}$ is a generating family of $\pi \circ i$, where $H_{0}^{\prime} \in \mathfrak{M}(r ; n)^{2}$ is defined by $H_{0}^{\prime}(x, y)=H_{0}(x, 0, y)$. Then there exists a quadratic form $T\left(Q_{r+1}, \ldots, Q_{n}, p_{1}, \ldots, p_{n}\right)$ such that the function germ $H_{0}^{\prime \prime}(Q, p)=H_{0}^{\prime}\left(Q_{1}, \ldots, Q_{r}, p\right)+T\left(Q_{r+1}, \ldots, Q_{n}, p\right)$ also defined the symplectic diffeomorphism $S_{H_{0}^{\prime \prime}}$. Then we have that $\left.\pi \circ S_{H_{0}^{\prime \prime}}\right|_{\mathbb{L}}=\pi \circ i$. Therefore we may assume that $H_{0}^{\prime \prime}=H_{0}$

We have that $H_{0}^{\prime}$ is stably reticular $\mathcal{R}$-equivalent to $F(x, y, 0)$. Therefore we need only to prove that $H_{0}^{\prime}$ is a simple singularity.

There exists a neighborhood $V$ of $j^{n+2} H_{0}^{\prime}(0)$ such that the map

$$
V \rightarrow S^{n+1}(n), j^{n+2} H^{\prime}(0) \mapsto j_{0}^{n+1} S_{H}(0),
$$

where $H(Q, p)=H^{\prime}\left(Q_{1}, \ldots, Q_{r}, p\right)+T\left(Q_{r+1}, \ldots, Q_{n}, p\right)$ is well defined and continuous.

Since $\pi \circ i$ is simple, there exist finite symplectic diffeomorphism germs $S_{1}, \ldots, S_{m}$ on $\left(T^{*} \mathbb{R}^{n}, 0\right)$ and a neighborhood $U_{z}$ of $z=j^{n+1} S_{0}(0)$ in $S^{n+1}(n)$ such that $U_{z} \subset$ $\left[S_{1}\right] \cup \cdots \cup\left[S_{m}\right]$ Let $V^{\prime}$ be the inverse image of $U_{z}$ by the above map. Then we have that $V^{\prime} \subset\left[z_{1}\right] \cup \cdots \cup\left[z_{m}\right]$, where $z_{j}=j^{n+2} H_{j}(0)$ for a function germ $H_{j}^{\prime} \in \mathcal{E}(r ; n)$ such that $j^{n+1} S_{j}(0)=j^{n+1} S_{H_{j}}(0)$. This means that $H_{0}^{\prime}$ is simple.

TheOREM 6.11. Let $r=0, n \leq 5$ or $r=1, n \leq 3$. Let $U$ be a neighborhood of 0 in $T^{*} \mathbb{R}^{n}$. Then there exists a residual set $O \subset S\left(U, T^{*} \mathbb{R}^{n}\right)$ such that for any $\tilde{S} \in O$ and $w=\left(0, \ldots, p_{r+1}^{0}, \ldots, p_{n}^{0}\right) \in U$, the reticular Lagrangian map $\left.\pi \circ \tilde{S}_{w}\right|_{\mathbb{L}}$ is stable.

In the case $r=0, n \leq 5$. A reticular Legendrian map $\left.\pi \circ \tilde{S}_{w}\right|_{\mathbb{L}}$ for any $\tilde{S} \in O$ and $w \in U$ has a generating family $F$ which is a reticular $\mathcal{P}$ - $\mathcal{R}^{+}$-stable unfolding of one of $A_{2}, A_{3}^{ \pm}, A_{4}, A_{5}^{ \pm}, A_{6}, D_{4}^{ \pm}, D_{4}^{ \pm}, D_{6}^{ \pm}, E_{6}^{ \pm}$, that is $F$ is stably reticular $\mathcal{P}$ - $\mathcal{R}^{+}$-equivalent to one of the following list:

$A_{2}: F\left(y_{1}, q_{1}\right)=y_{1}^{3}+q_{1} y_{1}$,

$A_{3}^{ \pm}: F\left(y_{1}, q_{1}, q_{2}\right)= \pm y_{1}^{4}+q_{1} y_{1}^{2}+q_{2} y_{1}$

$A_{4}: F\left(y_{1}, q_{1}, q_{2}, q_{3}\right)=y_{1}^{5}+q_{1} y_{1}^{3}+q_{2} y_{1}^{2}+q_{3} y_{1}$,

$A_{5}^{ \pm}: F\left(y_{1}, q_{1}, q_{2}, q_{3}, q_{4}\right)= \pm y_{1}^{6}+q_{1} y_{1}^{4}+q_{2} y_{1}^{3}+q_{3} y_{1}^{2}+q_{4} y_{1}$,

$A_{6}: F\left(y_{1}, q_{1}, q_{2}, q_{3}, q_{4}, q_{5}\right)=y_{1}^{7}+q_{1} y_{1}^{5}+q_{2} y_{1}^{4}+q_{3} y_{1}^{3}+q_{4} y_{1}^{2}+q_{5} y_{1}$,

$D_{4}^{ \pm}: F\left(y_{1}, y_{2}, q_{1}, q_{2}, q_{3}\right)=y_{1}^{2} y_{2} \pm y_{2}^{3}+q_{1} y_{2}^{2}+q_{2} y_{2}+q_{3} y_{1}$,

$D_{5}^{ \pm}: F\left(y_{1}, y_{2}, q_{1}, q_{2}, q_{3}, q_{4}\right)=y_{1}^{2} y_{2} \pm y_{2}^{4}+q_{1} y_{2}^{3}+q_{2} y_{2}^{2}+q_{3} y_{2}+q_{4} y_{1}$,

$D_{6}^{ \pm}: F\left(y_{1}, y_{2}, q_{1}, q_{2}, q_{3}, q_{4}, q_{5}\right)=y_{1}^{2} y_{2} \pm y_{2}^{5}+q_{1} y_{2}^{4}+q_{2} y_{2}^{3}+q_{3} y_{2}^{2}+q_{4} y_{2}+q_{5} y_{1}$,

$E_{6}^{ \pm}: F\left(y_{1}, y_{2}, q_{1}, q_{2}, q_{3}, q_{4}, q_{5}\right)=y_{1}^{3} \pm y_{2}^{4}+q_{1} y_{1} y_{2}^{2}+q_{2} y_{1} y_{2}+q_{3} y_{2}^{2}+q_{4} y_{1}+q_{5} y_{2}$.

In the case $r=1, n \leq 3$. A reticular Legendrian map $\left.\pi \circ \tilde{S}_{w}\right|_{\mathbb{L}}$ for any $\tilde{S} \in O$ and $w \in U$ has a generating family which is a $\mathcal{P}$ - $\mathcal{R}^{+}$-stable unfolding of one of $B_{2}^{ \pm}, B_{3}^{ \pm}, B_{4}^{ \pm}, C_{3}^{ \pm}, C_{4}^{ \pm}, F_{4}^{ \pm}$, that is $F$ is stably reticular $\mathcal{P}$ - $\mathcal{R}^{+}$-equivalent to one of the following list:

$B_{2}^{ \pm}: F\left(x, q_{1}\right)= \pm x^{2}+q_{1} x$,

$B_{3}^{ \pm}: F\left(x, q_{1}, q_{2}\right)= \pm x^{3}+q_{1} x^{2}+q_{2} x$,

$B_{4}^{ \pm}: F\left(x, q_{1}, q_{2}, q_{3}\right)= \pm x^{4}+q_{1} x^{3}+q_{2} x^{2}+q_{1} x$,

$C_{3}^{ \pm}: F\left(x, y, q_{1}, q_{2}\right)= \pm x y+y^{3}+q_{1} y^{2}+q_{2} y$,

$C_{4}^{ \pm}: F\left(x, y, q_{1}, q_{2}, q_{3}\right)= \pm x y+y^{4}+q_{1} y^{3}+q_{2} y^{2}+q_{3} y$,

$F_{4}^{ \pm}: F\left(x, y, q_{1}, q_{2}, q_{3}\right)= \pm x^{2}+y^{3}+q_{1} x y+q_{2} x+q_{3} y$.

Proof. We need only to prove the case $r=1, n \leq 3$. Let $F_{X}(x, y, q) \in \mathfrak{M}(r ; k+n)^{2}$ 
be a reticular $\mathcal{P}-\mathcal{R}^{+}$-stable unfolding of singularity $X \in \mathfrak{M}(r ; k)^{2}$ for

$$
X=B_{2}^{ \pm}, B_{3}^{ \pm}, B_{4}^{ \pm}, C_{3}^{ \pm}, C_{4}^{ \pm}, F_{4}^{ \pm} .
$$

Then other unfoldings are not stable since other singularities have reticular $\mathcal{R}^{+}$codimension $>3$. We choose stable reticular Lagrangian maps $\pi \circ i_{X}:(\mathbb{L}, 0) \rightarrow$ $\left(T^{*} \mathbb{R}^{n}, 0\right) \rightarrow\left(\mathbb{R}^{n}, 0\right)$ with generating families $F_{X}$ and $S_{X}$ be extensions of $i_{X}$ for above list. We set $L_{U}=\left\{(q, p) \in U \mid q=p_{1}=0\right\}$ and define that

$$
O^{\prime}=\left\{\tilde{S} \in S\left(U, T^{*} \mathbb{R}^{n}\right) \mid j_{0}^{n+1} \tilde{S} \text { is transversal to }\left[j^{n+1} S_{X}(0)\right] \text { on } L_{U} \text { for all } X\right\}
$$

Then $O^{\prime}$ is a residual set. We set

$$
Y=\left\{j^{n+1} S(0) \in S^{n+1}(n) \mid \text { the codimension of }\left[j^{n+1} S(0)\right]>2 n\right\} .
$$

Then $Y$ is an algebraic set in $S^{n+1}(n)$ by Theorem 6.6 (a'). Therefore we can define that

$$
O^{\prime \prime}=\left\{\tilde{S} \in S\left(U, T^{*} \mathbb{R}^{n}\right) \mid j_{0}^{n+1} \tilde{S} \text { is transversal to } Y\right\}
$$

Then $Y$ has codimension $>2 n$ because all element in $Y$ is adjacent to one of the above list which are simple. Then we have that

$$
O^{\prime \prime}=\left\{\tilde{S} \in S\left(U, T^{*} \mathbb{R}^{n}\right) \mid j_{0}^{n+1} \tilde{S}(U) \cap Y=\emptyset\right\} .
$$

We define $O=O^{\prime} \cap O^{\prime \prime}$. Then $O$ has the required condition.

In the case $r=0, n \leq 5$. Set $X=A_{2}, A_{3}^{ \pm}, A_{4}, A_{5}^{ \pm}, A_{6}, D_{4}^{ \pm}, D_{4}^{ \pm}, D_{6}^{ \pm}, E_{6}^{ \pm}$and $Y=\left\{j^{n+1} S(0) \in S^{n+1}(n) \mid\right.$ the codimension of $\left.\left[j^{n+1} S(0)\right]>2 n\right\}$. Then we have that the codimension of $Y$ in $S^{n+1}(n)$ is higher than $2 n$ and the assertion is proved by the parallel method of the above case.

7. Genericity of reticular Legendrian maps. Let $J^{l}(2 n+1,2 n+1)$ be the set of $l$-jets of map germs from $\left(J^{1}\left(\mathbb{R}^{n}, \mathbb{R}\right), 0\right)$ to $\left(J^{1}\left(\mathbb{R}^{n}, \mathbb{R}\right), 0\right)$ and $C^{l}(n)$ be the Lie group in $J^{l}(2 n+1,2 n+1)$ consists of $l$-jets of contact diffeomorphism germs on $\left(J^{1}\left(\mathbb{R}^{n}, \mathbb{R}\right), 0\right)$. We consider the Lie subgroup $r L e^{l}(n)$ of $L^{l}(2 n+1) \times L^{l}(2 n+1)$ consists of $l$-jets of reticular diffeomorphisms on the source space and $l$-jets of Legendrian equivalences of $\tilde{\pi}$ on the target space:

$$
r L e^{l}(n)=\left\{\left(j^{l} \phi(0), j^{l} \Theta(0)\right) \in L^{l}(2 n+1) \times L^{l}(2 n+1) \mid \phi\right. \text { is a reticular }
$$
diffeomorphism on $\left(J^{1}\left(\mathbb{R}^{n}, \mathbb{R}\right), 0\right), \Theta$ is a Legendrian equivalence of $\left.\tilde{\pi}\right\}$.

The group $r L e^{l}(n)$ acts on $J^{l}(2 n+1,2 n+1)$ and $C^{l}(n)$ is invariant under this action. Let $C$ be a contact diffeomorphism germ on $\left(J^{1}\left(\mathbb{R}^{n}, \mathbb{R}\right), 0\right)$ and set $z=j^{l} C(0)$. We denote the orbit $r L e^{l}(n) \cdot z$ by $[z]$. Then

$$
[z]=\left\{j^{l} C^{\prime}(0) \in C^{l}(n) \mid \tilde{\pi} \circ i \text { and }\left.\tilde{\pi} \circ C^{\prime}\right|_{\tilde{\mathbb{L}}} \text { are Legendrian equivalent }\right\} .
$$

In this section we denote by $X_{f}$ the Contact Hamiltonian vector field on $\left(J^{1}\left(\mathbb{R}^{n}, \mathbb{R}\right), 0\right)$ for a function germ $f$ on $\left(J^{1}\left(\mathbb{R}^{n}, \mathbb{R}\right), 0\right)$. That is

$$
X_{f}=\sum_{j=1}^{n}\left(\frac{\partial f}{\partial q_{j}}+p_{j} \frac{\partial f}{\partial z}\right) \frac{\partial}{\partial p_{j}}-\sum_{j=1}^{n} \frac{\partial f}{\partial p_{j}} \frac{\partial}{\partial q_{j}}+\left(f-\sum_{j=1}^{n} p_{j} \frac{\partial f}{\partial p_{j}}\right) \frac{\partial}{\partial z} .
$$






FIG. 2. the caustic $B_{3}^{ \pm}$
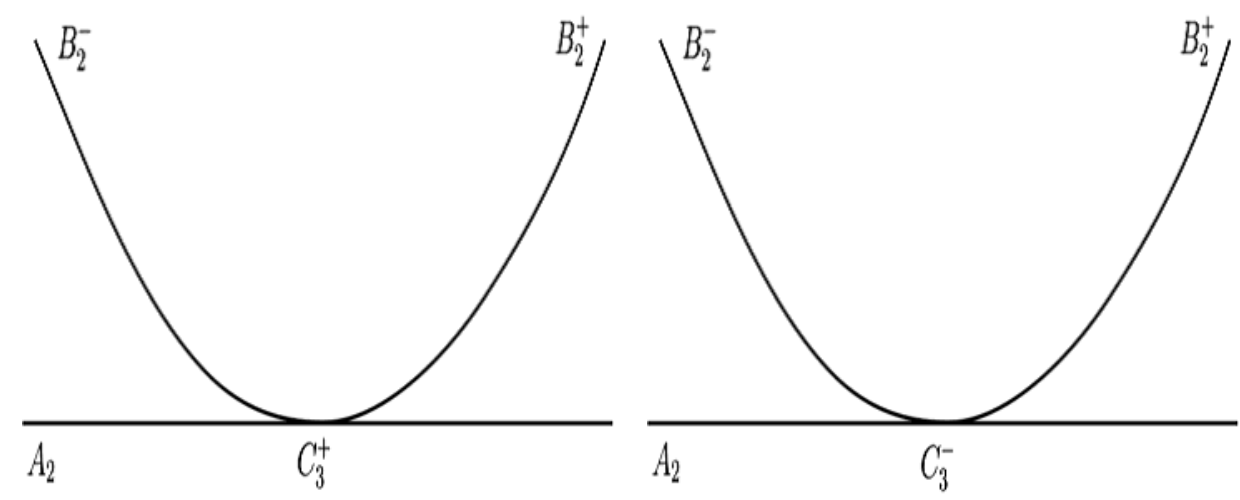

FIG. 3. the caustics $C_{3}^{ \pm}$

We denote by $V I_{C}$ the vector space consists of infinitesimal contact transformation germs of $C$ and denote by $V I_{C}^{0}$ the subspace of $V I_{C}$ consists of germs which vanish on 0 . We denote by $V L_{J^{1}\left(\mathbb{R}^{n}, \mathbb{R}\right)}$ by the vector space consists of infinitesimal Legendrian equivalences on $\left(J^{1}\left(\mathbb{R}^{n}, \mathbb{R}\right), 0\right)$ and denote by $V L_{J^{1}\left(\mathbb{R}^{n}, \mathbb{R}\right)}^{0}$ by the subspace of $V L_{J^{1}\left(\mathbb{R}^{n}, \mathbb{R}\right)}$ consists of germs which vanish at 0 . We denote by $V_{\tilde{\mathbb{L}}}^{0}$ the vector space consists of infinitesimal reticular diffeomorphisms on $\left(J^{1}\left(\mathbb{R}^{n}, \mathbb{R}\right), 0\right)$ which vanishes at 0 :

$$
V_{\tilde{\mathbb{L}}}^{0}=\left\{\xi \in X\left(J^{1}\left(\mathbb{R}^{n}, \mathbb{R}\right), 0\right) \mid \xi \text { is tangent to } \tilde{L}_{\sigma}^{0} \text { for all } \sigma \subset I_{r}, \xi(0)=0\right\}
$$

Lemma 7.1. (1) A vector field germ $v$ on $\left(J^{1}\left(\mathbb{R}^{n}, \mathbb{R}\right), 0\right)$ along $C$ belongs to $V I_{C}$ if and only if there exists a function germ $f$ on $\left(J^{1}\left(\mathbb{R}^{n}, \mathbb{R}\right), 0\right)$ such that $v=X_{f} \circ C$, (2) A vector field germ $\eta$ on $\left(J^{1}\left(\mathbb{R}^{n}, \mathbb{R}\right), 0\right)$ belongs to $V L_{J^{1}\left(\mathbb{R}^{n}, \mathbb{R}\right)}$ if and only if there exists a fiber preserving function germ $H$ on $\left(J^{1}\left(\mathbb{R}^{n}, \mathbb{R}\right), 0\right)$ such that $\eta=X_{H}$.

(3) A vector field germ $\xi$ on $\left(J^{1}\left(\mathbb{R}^{n}, \mathbb{R}\right), 0\right)$ belongs to $V_{\tilde{\mathbb{L}}}^{0}$ if and only if there exists 

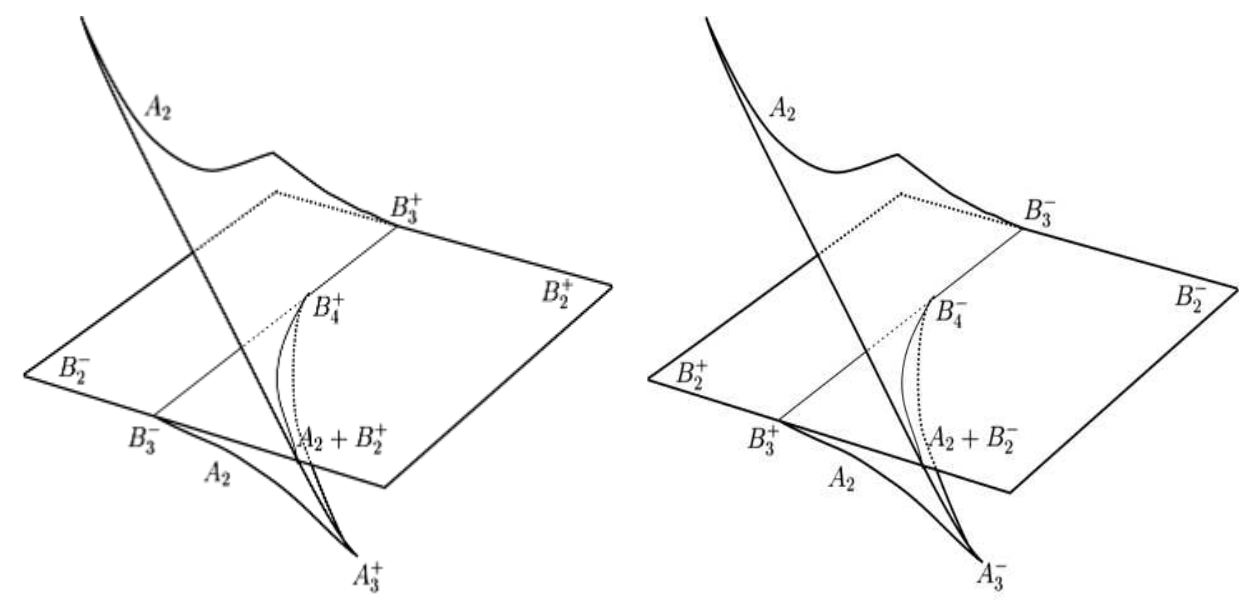

FIG. 4. the caustics $B_{4}^{ \pm}$
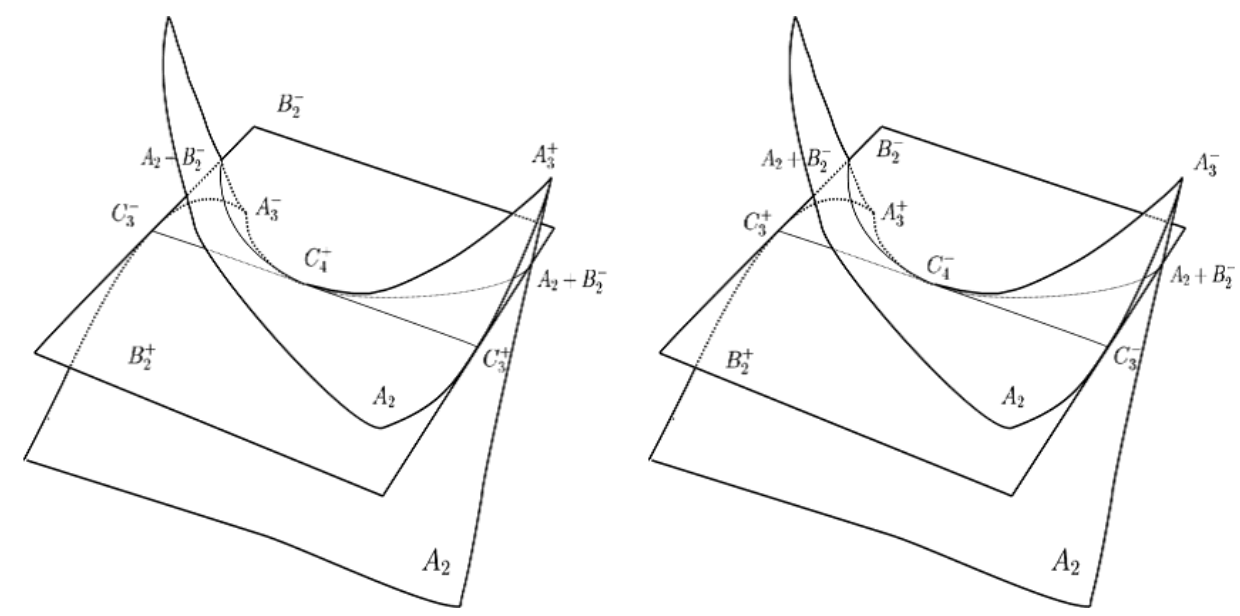

FIG. 5. the caustics $C_{4}^{ \pm}$

a function germ $g \in B_{0}^{\prime}$ such that $\xi=X_{g}$, where $B_{0}^{\prime}=\left\langle q_{1} p_{1}, \ldots, q_{r} p_{r}\right\rangle_{\mathcal{E}_{J^{1}\left(\mathbb{R}^{n}, \mathbb{R}\right)}}+$ $\mathfrak{M}_{J^{1}\left(\mathbb{R}^{n}, \mathbb{R}\right)}\left\langle q_{r+1}, \ldots, q_{n}, z\right\rangle$ is a submodule of $\mathcal{E}_{J^{1}\left(\mathbb{R}^{n}, \mathbb{R}\right)}$

By this lemma we have that:

$V I_{C}^{0}=\left\{v:\left(J^{1}\left(\mathbb{R}^{n}, \mathbb{R}\right), 0\right) \rightarrow\left(T\left(J^{1}\left(\mathbb{R}^{n}, \mathbb{R}\right)\right), 0\right) \mid v=X_{f} \circ C\right.$ for some $\left.f \in \mathfrak{M}_{J^{1}\left(\mathbb{R}^{n}, \mathbb{R}\right)}^{2}\right\}$,

$V L_{J^{1}\left(\mathbb{R}^{n}, \mathbb{R}\right)}^{0}=\left\{\eta \in X\left(J^{1}\left(\mathbb{R}^{n}, \mathbb{R}\right), 0\right) \mid \eta=X_{H}\right.$

for some fiver preserving function germ $\left.H \in \mathfrak{M}_{J^{1}\left(\mathbb{R}^{n}, \mathbb{R}\right)}^{2}\right\}$,

$V_{\tilde{\mathbb{L}}}^{0}=\left\{\xi \in X\left(J^{1}\left(\mathbb{R}^{n}, \mathbb{R}\right), 0\right) \mid \xi=X_{g}\right.$ for some $\left.g \in B_{0}^{\prime}\right\}$. 

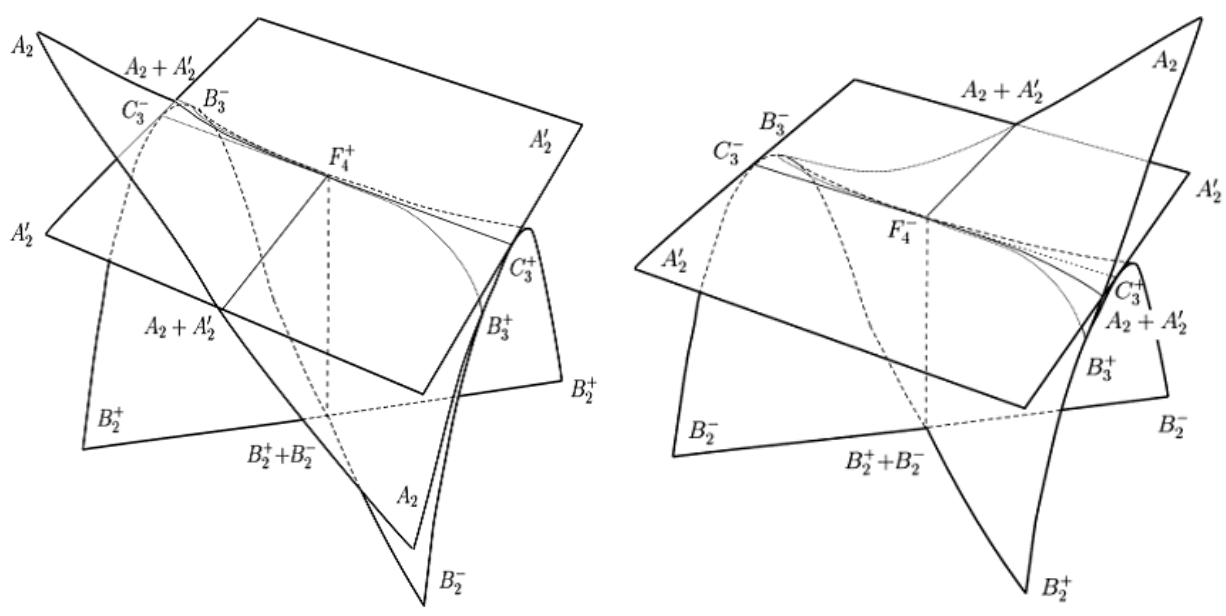

FIG. 6. the caustics $F_{4}^{ \pm}$

We define the homomorphism $t C: V_{\tilde{\mathbb{L}}}^{0} \rightarrow V I_{C}^{0}$ by $t C(v)=C_{*} v$ and define the homomorphism $w C: V L_{J^{1}\left(\mathbb{R}^{n}, \mathbb{R}\right)}^{0} \rightarrow V I_{C}^{0}$ by $w C(\eta)=\eta \circ C$.

We denote $V I_{C}^{l}$ the subspace of $V I_{C}$ consists of infinitesimal contact transformation germs of $C$ whose $l$-jets are 0 :

$$
V I_{C}^{l}=\left\{v \in V I_{C} \mid j^{l} v(0)=0\right\} .
$$

We have that $j_{0}^{l} C$ is transversal to $[z]$ if and only if

$$
\left.t C\left(V_{\tilde{\mathbb{L}}}^{0}\right)+w C\left(V L_{J^{1}\left(\mathbb{R}^{n}, \mathbb{R}\right)}\right)\right)+V I_{C}^{l+1}=V I_{C} .
$$

Let $N, M$ be $(2 n+1)$-dimensional contact manifolds. We denote $C(N, M)$ the space of contact embeddings from $N$ to $M$ with the topology induced by the Whitney $C^{\infty}$-topology of $C^{\infty}(N, M)$ and define that

$$
\begin{aligned}
J_{C}^{l}(N, M)= & \left\{j^{l} C\left(u_{0}\right) \in J^{l}(N, M) \mid C:\left(N, u_{0}\right)\right. \\
& \left.\rightarrow M \text { is a contact embedding germ, } u_{0} \in N\right\} .
\end{aligned}
$$

Proposition 7.2. $C(N, M)$ is a Baire space.

The following theorem is the spacial case of [6, p.156 Proposition 9.5].

Theorem 7.3. Let $N, M$ be $(2 n+1)$-dimensional contact manifolds. Let $Q_{j}, j=$ $1,2, \ldots$ be submanifolds of $J_{C}^{l}(N, M)$. Then the set

$$
T=\left\{C \in C(N, M) \mid j^{l} C \text { is transversal to } Q_{j} \text { for all } j \in \mathbb{N}\right\}
$$

is residual set in $C(N, M)$. In particular $T$ is dense. 
TheOREM 7.4. Let $U$ be a neighborhood 0 in $J^{1}\left(\mathbb{R}^{n}, \mathbb{R}\right), Q_{1}, Q_{2}, \ldots$ are submanifolds of $C^{l}(n)$. We define $L_{U}=\left\{(q, z, p) \in U \mid q=z=p_{1}=\cdots=p_{r}=0\right\}$ Then the set

$$
T=\left\{\tilde{C} \in C^{\theta}\left(U, J^{1}\left(\mathbb{R}^{n}, \mathbb{R}\right)\right) \mid j_{0}^{l} \tilde{C} \text { is transversal to } Q_{j} \text { on } L_{U} \text { for all } j\right\}
$$

is a residual set in $C^{\theta}\left(U, J^{1}\left(\mathbb{R}^{n}, \mathbb{R}\right)\right)$.

We have the following theorem which is proved by a parallel method of Theorem 6.6

TheOREM 7.5. Let $\tilde{\pi} \circ i:(\tilde{\mathbb{L}}, 0) \rightarrow\left(J^{1}\left(\mathbb{R}^{n}, \mathbb{R}\right), 0\right) \rightarrow\left(\mathbb{R}^{n} \times \mathbb{R}, 0\right)$ be a reticular Legendrian map. Let $C$ be an extension of $i$ and $l \geq n+2$. Let $B^{\prime}=$ $\left\langle q_{1} p_{1}, \ldots, q_{r} p_{r}, q_{r+1}, \ldots, q_{n}, z\right\rangle_{\mathcal{E}_{J^{1}\left(\mathbb{R}^{n}, \mathbb{R}\right)}}$ be a submodule of $\mathcal{E}_{J^{1}\left(\mathbb{R}^{n}, \mathbb{R}\right)}$. Then the following are all equivalent:

(s) $\tilde{\pi} \circ i$ is stable.

(t) $j_{0}^{l} C$ is transversal to $\left[j_{0}^{l} C(0)\right]$.

(a') $\mathcal{E}_{J^{1}\left(\mathbb{R}^{n}, \mathbb{R}\right)} /\left(B^{\prime}+\mathfrak{M}_{J^{1}\left(\mathbb{R}^{n}, \mathbb{R}\right)}^{l+2}\right)$ is generated by $1, p_{1} \circ C, \ldots, p_{n} \circ C$ as an $\mathcal{E}_{q, z^{-}}$-module via $(\tilde{\pi} \circ C)^{*}$.

(a") $\mathcal{E}_{J^{1}\left(\mathbb{R}^{n}, \mathbb{R}\right)} / B^{\prime}$ is generated by $1, p_{1} \circ C, \ldots, p_{n} \circ C$ as an $\mathcal{E}_{q, z}$-module via $(\tilde{\pi} \circ C)^{*}$. (is) $\tilde{\pi} \circ i$ is infinitesimally stable.

For $\tilde{C} \in C^{\theta}\left(U, J^{1}\left(\mathbb{R}^{n}, \mathbb{R}\right)\right)$ we define the continuous map $j_{0}^{l} \tilde{C}: U \rightarrow C^{l}(n)$ by $w=\left(q_{0}, z_{0}, p_{0}\right)$ to the $l$-jet of $\tilde{C}_{w}$ at 0 , where the contact diffeomorphism germ $\tilde{C}_{w}$ on $\left(J^{1}\left(\mathbb{R}^{n}, \mathbb{R}\right), 0\right)$ is given by

$$
x=(q, z, p) \mapsto C(w+x)-C(w)+\left(0,-p_{C}(w) q_{C}(w+x)+p_{C}(w) q_{C}(w)+p_{0} q, 0\right) .
$$

Let $\tilde{\pi} \circ i:(\tilde{\mathbb{L}}, 0) \rightarrow\left(J^{1}\left(\mathbb{R}^{n}, \mathbb{R}\right), 0\right) \rightarrow C^{\theta}\left(\mathbb{R}^{n} \times \mathbb{R}, 0\right)$ be a stable reticular Legendrian map. We say that $\tilde{\pi} \circ i$ is simple if there exists a representative $\tilde{C} \in C^{\theta}\left(U, J^{1}\left(\mathbb{R}^{n}, \mathbb{R}\right)\right)$ of a extension of $i$ such that $\left\{\tilde{C}_{w} \mid w \in U\right\}$ is covered by finite orbits $\left[C_{1}\right], \ldots,\left[C_{m}\right]$ for some contact diffeomorphism germs $C_{1}, \ldots, C_{m}$ on $\left(J^{1}\left(\mathbb{R}^{n}, \mathbb{R}\right), 0\right)$.

By an analogous method of Lemma 6.9, Lemma 6.10 and Theorem 6.11 we have the followings:

Lemma 7.6. Let $\tilde{\pi} \circ i:(\tilde{\mathbb{L}}, 0) \rightarrow\left(J^{1}\left(\mathbb{R}^{n}, \mathbb{R}\right), 0\right) \rightarrow\left(\mathbb{R}^{n} \times \mathbb{R}, 0\right)$ be a stable reticular Legendrian map. Then $\tilde{\pi} \circ i$ is simple if and only if there exist a neighborhood $U_{z}$ of $z=j_{0}^{n+3} C(0)$ in $C^{n+3}(n)$ and $z_{1}, \ldots, z_{m} \in C^{n+3}(n)$ such that $U_{z} \subset\left[z_{1}\right] \cup \cdots \cup\left[z_{m}\right]$.

Lemma 7.7. A stable reticular Legendrian map $\tilde{\pi} \circ i$ is simple if and only if for a generating family $F(x, y, q, z) \in \mathfrak{M}(r ; k+n+1)$ of $\tilde{\pi} \circ i, F(x, y, 0,0) \in \mathfrak{M}(r ; k)^{2}$ is $\mathcal{K}$-simple singularity.

THEOREM 7.8. Let $r=0, n \leq 6$ or $r=1, n \leq 4$. Let $U$ be a neighborhood of 0 in $J^{1}\left(\mathbb{R}^{n}, \mathbb{R}\right)$. Then there exists a residual set $O \subset C^{\theta}\left(U, J^{1}\left(\mathbb{R}^{n}, \mathbb{R}\right)\right)$ such that for any $\tilde{C} \in O$ and $w=\left(0, \ldots, 0, p_{r+1}, \ldots, p_{n}\right) \in U$, the reticular Legendrian map $\left.\tilde{\pi} \circ \tilde{C}_{w}\right|_{\tilde{\mathbb{L}}}$ is stable.

In the case $r=0, n \leq 6$. A reticular Legendrian map $\left.\tilde{\pi} \circ \tilde{C}_{w}\right|_{\tilde{\mathbb{L}}}$ for any $\tilde{C} \in O$ and $w \in U$ has a generating family which is a reticular $\mathcal{P}-\mathcal{K}$-stable unfolding of one of $A_{1}, A_{2}, A_{3}, A_{4}, A_{4}, A_{6}, D_{4}^{ \pm}, D_{5}, D_{6}^{ \pm}, E_{6}$, that is $F$ is stably reticular $\mathcal{P}$ - $\mathcal{K}$-equivalent to one of the following list: 
$A_{2}: F\left(y_{1}, z\right)=y_{1}^{2}+z$,

$A_{2}: F\left(y_{1}, q_{1}, z\right)=y_{1}^{3}+q_{1} y_{1}+z$,

$A_{3}: F\left(y_{1}, q_{1}, q_{2}, z\right)=y_{1}^{4}+q_{1} y_{1}^{2}+q_{2} y_{1}+z$,

$A_{4}: F\left(y_{1}, q_{1}, q_{2}, q_{3}\right)=y_{1}^{5}+q_{1} y_{1}^{3}+q_{2} y_{1}^{2}+q_{3} y_{1}+z$,

$A_{5}: F\left(y_{1}, q_{1}, q_{2}, q_{3}, q_{4}\right)=y_{1}^{6}+q_{1} y_{1}^{4}+q_{2} y_{1}^{3}+q_{3} y_{1}^{2}+q_{4} y_{1}+z$,

$A_{6}: F\left(y_{1}, q_{1}, q_{2}, q_{3}, q_{4}, q_{5}, z\right)=y_{1}^{7}+q_{1} y_{1}^{5}+q_{2} y_{1}^{4}+q_{3} y_{1}^{3}+q_{4} y_{1}^{2}+q_{5} y_{1}+z$,

$D_{4}^{ \pm}: F\left(y_{1}, y_{2}, q_{1}, q_{2}, q_{3}, z\right)=y_{1}^{2} y_{2} \pm y_{2}^{3}+q_{1} y_{2}^{2}+q_{2} y_{2}+q_{3} y_{1}+z$,

$D_{5}: F\left(y_{1}, y_{2}, q_{1}, q_{2}, q_{3}, q_{4}, z\right)=y_{1}^{2} y_{2}+y_{2}^{4}+q_{1} y_{2}^{3}+q_{2} y_{2}^{2}+q_{3} y_{2}+q_{4} y_{1}+z$,

$D_{6}^{ \pm}: F\left(y_{1}, y_{2}, q_{1}, q_{2}, q_{3}, q_{4}, q_{5}, z\right)=y_{1}^{2} y_{2} \pm y_{2}^{5}+q_{1} y_{2}^{4}+q_{2} y_{2}^{3}+q_{3} y_{2}^{2}+q_{4} y_{2}+q_{5} y_{1}+z$,

$E_{6}: F\left(y_{1}, y_{2}, q_{1}, q_{2}, q_{3}, q_{4}, q_{5}, z\right)=y_{1}^{3}+y_{2}^{4}+q_{1} y_{1} y_{2}^{2}+q_{2} y_{1} y_{2}+q_{3} y_{2}^{2}+q_{4} y_{1}+q_{5} y_{2}+z$.

In the case $r=1, n \leq 4$. A reticular Legendrian map $\left.\tilde{\pi} \circ \tilde{C}_{w}\right|_{\tilde{\mathbb{L}}}$ for any $\tilde{C} \in O$ and $w \in U$ has a generating family which is a reticular $\mathcal{P}$ - $\mathcal{K}$-stable unfolding of one of $B_{2}, B_{3}, B_{4}, C_{3}^{ \pm}, C_{4}, F_{4}$, that is $F$ is stably reticular $\mathcal{P}$ - $\mathcal{K}$-equivalent to one of the following list:

$B_{2}: F\left(x, q_{1}, z\right)=x^{2}+q_{1} x+z$,

$B_{3}: F\left(x, q_{1}, q_{2}, z\right)=x^{3}+q_{1} x^{2}+q_{2} x+z$,

$B_{4}: F\left(x, q_{1}, q_{2}, q_{3}, z\right)=x^{4}+q_{1} x^{3}+q_{2} x^{2}+q_{1} x+z$,

$C_{3}^{ \pm}: F\left(x, y, q_{1}, q_{2}, z\right)= \pm x y+y^{3}+q_{1} y^{2}+q_{2} y+z$,

$C_{4}: F\left(x, y, q_{1}, q_{2}, q_{3}, z\right)=x y+y^{4}+q_{1} y^{3}+q_{2} y^{2}+q_{3} y+z$,

$F_{4}: F\left(x, y, q_{1}, q_{2}, q_{3}, z\right)=x^{2}+y^{3}+q_{1} x y+q_{2} x+q_{3} y+z$.

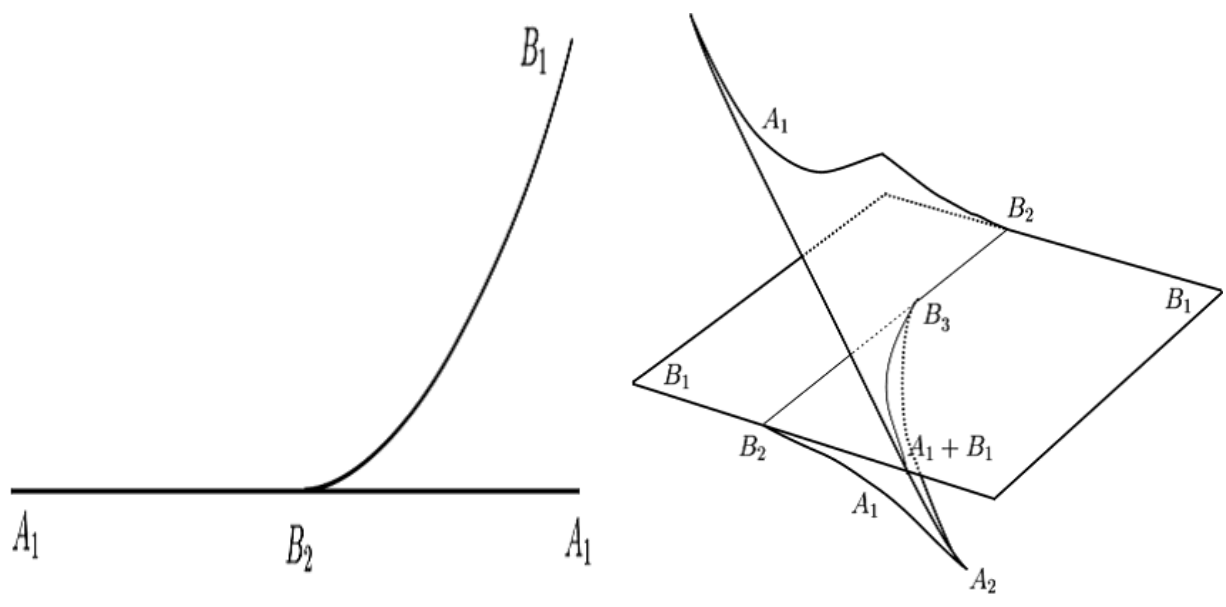

FIG. 7. the wavefronts $B_{2}$ and $B_{3}$

8. Classifications for the cases $r \geq 2$. In order to classify generic caustics for the case $r=2$, we can not use our equivalence relation. Caustic equivalence is still too strong. For example, consider the caustics with the generating families: $F_{a}\left(x_{1}, x_{2}, q_{1}, q_{2}\right)=x_{1}^{2}+x_{1} x_{2}+a x_{2}^{2}+q_{1} x_{1}+q_{2} x_{2}$. In these figures $Q_{1, I}, Q_{2, I}, Q_{e, 2}$ are in the same positions. Suppose that there exists a diffeomorphism germ $g$ on $\left(\mathbb{R}^{2}, 0\right)$ such that $Q_{1, I}, Q_{2, I}, Q_{e, 2}$ are invariant under $g$. Then $g$ can not map $Q_{e, 1}$ from one to the other. We need to admit that the above caustics are equivalent for some equivalence relation which is weaker than the caustic equivalence. We suggest 

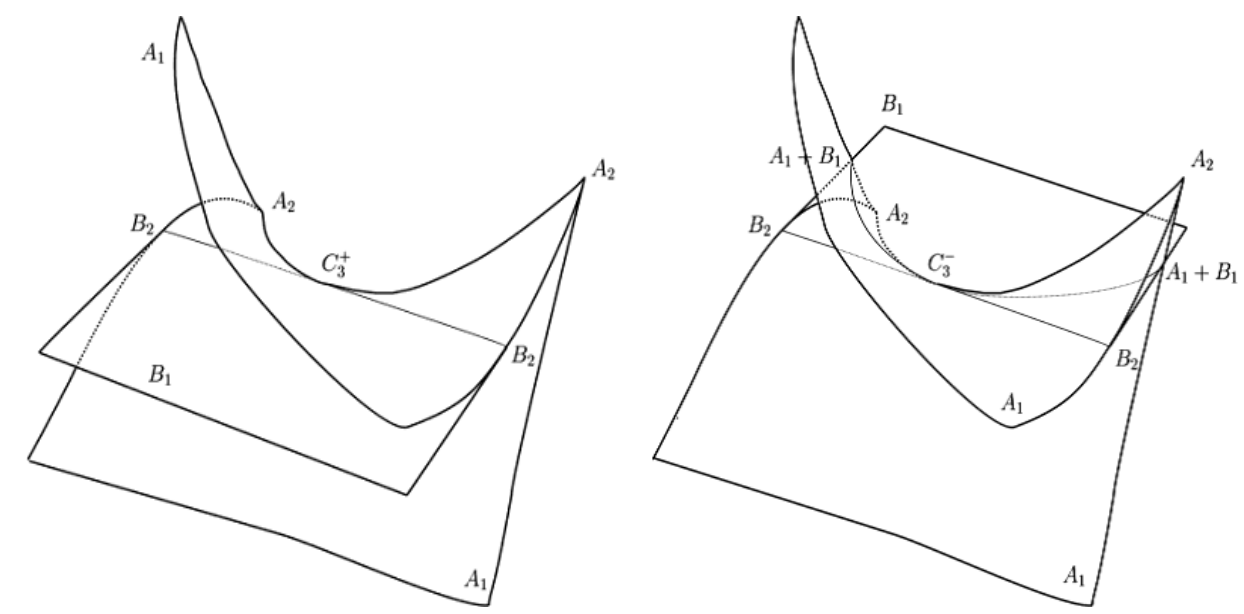

FIG. 8. the wavefronts $C_{3}^{ \pm}$
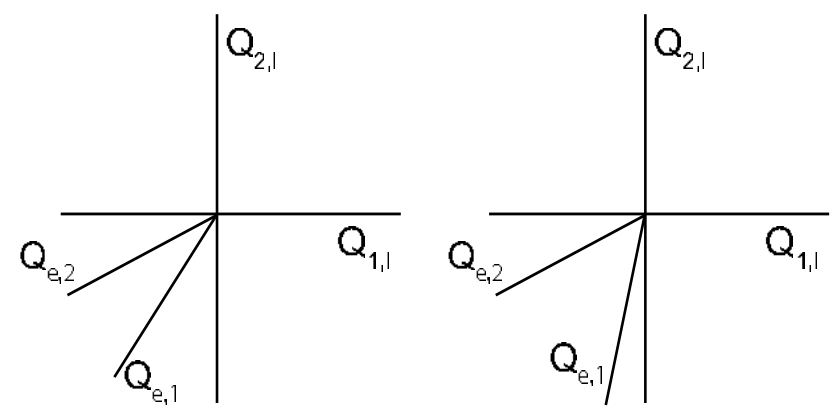

FIG. 9. the caustics of $F_{a}$ and $F_{b}\left(\frac{1}{4}<a<b, I=I_{2}, e=\emptyset\right)$

the equivalence relation consists on homeomorphism on $\left(\mathbb{R}^{n}, 0\right)$ which are smooth on and preserves all $Q_{\tau, \sigma}$ 's and $C_{\sigma}$ 's. The above two caustics are equivalent under this equivalence relation.

\section{REFERENCES}

[1] V.I. ARNOL'D, Normal forms for functions near degenerate critical points, the Weyl groups of $A_{k}, D_{k}, E_{k}$ and Lagrangian singularities, Functional Analysis and its applications, 6:4 (1972), pp. 254-272.

[2] V.I. Arnol'D, S.M. Gusuein-Zade, and A.N. Varchenko, Singularities of Differentiable Maps I, Birkhauser, 1985.

[3] M. Golubitsky and V. Guillemin, Stable mappings and their singularities, Graduate Texts in Mathematics, 1973.

[4] G. Ishikawa, Transversalities for Lagrange singularities of isotropic mapping of corank one, Banach Center Publ., 33 (1996), pp. 93-104.

[5] G. Ishikawa, Determinacy, transversality and Lagrange stability, Banach Center Publ., 50 (1999), pp. 123-135.

[6] G. IshiKaWA, Infinitesimal deformations and stability of singular Legendre submanifolds, Asian 
J. Math., 9:1 (2005), pp. 133-166.

[7] S. Janeczko, Generalized Luneburg canonical varieties and vector fields on quasicaustics, Journal of Mathematical Physics, 31 (1990), pp. 997-1009.

[8] I. Scherback, Boundary Fronts and Caustics and their Metamorphoses, Singularities: Lille, 1991, page 363, 1994.

[9] T. TsukAda, Reticular Lagrangian Singularities, Asian J. Math., 1 (1997), pp. 572-622.

[10] T. Tsukada, Reticular Legendrian Singularities, Asian J. Math., 5:1 (2001), pp. 109-127. 
T. TSUKADA 